\title{
Oblique Radial Glial Divisions in the Developing Mouse Neocortex Induce Self-Renewing Progenitors outside the Germinal Zone That Resemble Primate Outer Subventricular Zone Progenitors
}

\author{
Atsunori Shitamukai, Daijiro Konno, and Fumio Matsuzaki \\ RIKEN Center for Developmental Biology, Chuo-ku, Kobe 650-0047, Japan
}

\begin{abstract}
Radial glia cells function as neural stem cells in the developing brain and generate self-renewing and differentiating daughter cells by asymmetric cell divisions. During these divisions, the apical process or basal process of the elongated epithelial structure is asymmetrically partitioned into daughter cells, depending on developmental contexts. However, in mammalian neurogenesis, the relationship between these subcellular structures and self-renewability is largely unknown. We induced oblique cleavages of radial glia cells to split the apical and basal processes into two daughters, and investigated the fate and morphology of the daughters in slice cultures. We observed that the more basal daughter cell that inherits the basal process self-renews outside of the ventricular zone (VZ), while the more apical daughter cell differentiates. These self-renewing progenitors, termed "outer VZ progenitors," retain the basal but not the apical process, as recently reported for the outer subventricular zone (OSVZ) progenitors in primates (Fietz et al., 2010; Hansen et al., 2010); to selfrenew, they require clonal Notch signaling between sibling cells. We also found a small endogenous population of outer VZ progenitors in the mouse embryonic neocortex, consistent with a low frequency of oblique radial glia divisions. Our results describe the general role of the basal process in the self-renewal of neural progenitors and implicate the loss of the apical junctions during oblique divisions as a possible mechanism for generating OSVZ progenitors. We propose that mouse outer VZ progenitors, induced by oblique cleavages, provide a model to study both progenitor self-renewal and OSVZ progenitors.
\end{abstract}

\section{Introduction}

In mammalian brain development, highly elongated neuroepithelial cells, known as radial glia cells, undergo dynamic interkinetic nuclear migration (INM) and function as neural stem cells that generate both self-renewing progenitors and neurons (or committed intermediate progenitors). Therefore, the mechanisms underlying asymmetric division of radial glia cells are fundamental to brain development.

Studies of invertebrate models have demonstrated that typical modes of asymmetric cell division rely either on cell divisions that are oriented along the axis of cell polarity that asymmetrically localize cell fate determinants or along extrinsic signals from the microenvironment (niche) that promote self-renewal (for review, see Knoblich, 2008). Molecular machineries are evolutionarily conserved for the creation of cell polarity (e.g., the PAR complex that is composed of the Par3/Par6/atypical PKC proteins) and for orientation of the cleavage plane (e.g., the protein

Received Sept. 7, 2010; revised Dec. 30, 2010; accepted Jan. 14, 2011.

This study was partially supported by Grant-in-Aid for Scientific Research on Priority Areas from the Ministry of Education, Culture, Sports, Science and Technology-Japan. We thank G. Shioi and Matsuzaki laboratory members for discussions.

Correspondence should be addressed to Fumio Matsuzaki, RIKEN Center for Developmental Biology, 2-2-3 Minatojima Minamimachi, Chuo-ku, Kobe 650-0047 Japan. E-mail: fumio@cdb.riken.jp.

DOI:10.1523/JNEUROSCI.4773-10.2011

Copyright $\odot 2011$ the authors $\quad 0270-6474 / 11 / 313683-13 \$ 15.00 / 0$ complex containing the G $\alpha$ subunit and GoLoco motif G-protein regulator, such as LGN) (Manabe et al., 2002; Morin et al., 2007; Konno et al., 2008).

Time-lapse imaging studies have revealed that self-renewing progenitors in the developing rodent neocortex retain a radial fiber, an epithelial structure with a radial morphology, which is subdivided into the apical and basal processes by the cell body undergoing interkinetic nuclear migration (Hartfuss et al., 2001; Miyata et al., 2001, 2004; Noctor et al., 2001, 2004). Therefore, the epithelial structure has been implicated in neural cell fate determination. In this context, it has been proposed that the apical domain containing the PAR complex is involved in progenitor self-renewal (Kosodo et al., 2004; Costa et al., 2008; Bultje et al., 2009). However, recent studies indicate that the apical domain is essentially partitioned into both daughter cells in the majority of progenitor divisions during the mid-neurogenic stage in the mouse dorsal forebrain (Konno et al., 2008). Recent studies also demonstrate that a distinct type of self-renewing progenitor [i.e., the outer subventricular zone (OSVZ) progenitors] found in gyrencephala, such as primates and ferrets, retain only the basal process without the apical process (Fietz et al., 2010; Hansen et al., 2010). Thus, the role of the epithelial structure (polarity) in the inheritance of intrinsic or extrinsic determinants is still ambiguous.

Our previous study demonstrated that the more apical daughter cell assumes the differentiating cell fate when radial glia cells 
undergo oblique divisions. A recent study on neural progenitors in the zebrafish hindbrain indicated that this is indeed the case for their divisions that are oblique relative to the apical surface (Alexandre et al., 2010). Here, using time-lapse imaging of slice cultures, we addressed the relationship between the epithelial structure and progenitor self-renewal. We perturbed spindle orientation by disrupting LGN function to induce oblique radial glial divisions, which enabled us to separately analyze the role of the apical domain and basal process of the epithelial structure in cell fate determination. We found that self-renewing progenitors, which are generated by this process, are very similar to gyrencephalic OSVZ progenitors and are endogenously present as a minor population in the mouse neocortex.

\section{Materials and Methods}

Animals. All experiments used ICR mice, and embryonic stages were calculated using noon on the day of the vaginal plug as embryonic day 0.5 (E0.5). The research was performed in compliance with the guidelines for animal experiments at the RIKEN Center for Developmental Biology.

In utero electroporation and plasmids. In utero electroporation was performed as described previously (Konno et al., 2008). The loxP-polyAloxP (FloxP) conditional expression vector was constructed as follows: the loxP site was attached to the synthesized DNA oligo, and the PCRgenerated rabbit $\beta$-globin intron polyA signal was ligated into the NheI site of the pCAG-enhanced green fluorescent protein (EGFP)-N1 vector, as described previously (Konno et al., 2008; Kato et al., 2010). Based on this vector, we generated expression vectors with the CAG promoter or FloxP conditional expression vectors with the multicloning site of pEGFP-C1 or pEGFP-N1 (TAKARA Bio/Clontech) by replacing the EGFP with the PCR-generated monomeric Kusabira Orange (mKO) 1 and 2 or synthesized DNA oligo with the FLAG sequence. The $\mathrm{mKO} 2$ and $\mathrm{mKO} 1$ cDNA were purchased from MBL. mKO2-F was generated by PCR using the farnesylation signal (derived from pEGFP-F, TAKARA Bio) attached to long, synthesized oligo primers. The PCR-generated Cre recombinase (Cre), Notch intracellular domain (NICD; i.e., intracellular region of mouse Notch1 protein lacking C terminus PEST domain, 1744-2457 aa), LGN C terminus (LGN-C; i.e., LGN C terminus region of mouse LGN protein, 365-679 aa), and dominant-negative (DN)-Mindbomb-1 (Mib1; i.e., mouse Mindbomb-1 protein lacking $\mathrm{C}$ terminus ring finger domain, 1-767 aa) sequences were ligated into the CAG or FloxP expression vectors. All inserted DNA fragments that were generated by PCR were verified by DNA sequencing. The following plasmids were used in this study: pCAG-FloxpEGFP-N1, pCAG-Floxp-EGFP-F, pCAG-Floxp-mKO2-F, pCAG-FloxpFLAG-C1, pCAG-Floxp-FLAG-NICD, pCAG-Floxp-FLAG-LGN-C, pCAG-Floxp-DN-Mib1, pCAG-Cre, pCAG-PACT-mKO1, pCAG-mKO1ZO1, pCAG-Par3-EGFP, pCAG-EGFP-ZO1, pCAG-EGFP-3NLS, pCAG6Myc-LGN-C, and pCAG-DN-Mib1.

RNA interference experiments. Knockdown of LGN was performed as described previously [small interfering RNA (siRNA) for LGN] (Konno et al., 2008). The brains were electroplated with LGN siRNA (Stealth RNAi, Invitrogen) and pCAG-Cre and pCAG-Floxp-EGFP-N1 at E13.5, and were analyzed $48 \mathrm{~h}$ later.

Slice culture and time-lapse imaging. The basic technique of time-lapse imaging of brain slices was used as described previously (Miyata et al., 2001; Konno et al., 2008). To monitor the behavior and morphology of a clonal cell, we used a conditional expression vector system as described previously (Morin et al., 2007; Kato et al., 2010). The brains were coelectroporated with pCAG-Floxp conditional expression plasmids $(0.5$ $\mu \mathrm{g} / \mu \mathrm{l}$ EGFP and mKO2-F; $1.5 \mu \mathrm{g} / \mu \mathrm{l}$ empty vector, LGN-C, DN-Mib1, or NICD) and a low concentration of Cre recombinase expression vector $(0.015 \mathrm{ng} / \mu \mathrm{l})$, which resulted in high and dispersed gene expression (3-10 cells per slice). The electroporated embryos (E13.5 or E14.5) were dissected $24 \mathrm{~h}$ later in ice-cold DMEM/F-12 medium, and slices of dorsal cortex ( 150 to $300 \mu \mathrm{m}$ thick) were cut using an ophthalmic microsurgical knife (Alcon). Slices (on average, 20 ) were immersed in $\sim 130 \mu$ l of type Ia collagen (Cellmatrix, Nitta Gelatin) (diluted to $1.5 \mathrm{mg} / \mathrm{ml}$ with DMEM/F-12 and neutralizing buffer according to the manufacturer's protocol and kept on ice until use) at room temperature and then transferred onto a circular $30 \mathrm{~mm}$ membrane filter (Millipore). After a $15 \mathrm{~min}$ incubation at $37^{\circ} \mathrm{C}$, the slices were cultured at $37^{\circ} \mathrm{C}$ in $2-3 \mathrm{ml}$ of culture medium. Slice culture medium consisted of DMEM/F-12, 5\% horse serum (v/v), 5\% fetal bovine serum (v/v), N2 supplement (1:100, Invitrogen), B27 supplement without retinoic acid (1:50, Invitrogen), $100 \mathrm{U} / \mathrm{ml}$ penicillin/streptomycin, $10 \mathrm{ng} / \mathrm{ml}$ epidermal growth factor, and $10 \mathrm{ng} / \mathrm{ml}$ basic fibroblast growth factor. The POC-Chamber-System (Olympus) was filled with $40 \% \mathrm{O}_{2}, 5 \% \mathrm{CO}_{2}$, and $55 \% \mathrm{~N}_{2}$ within the incubation chamber. The sample was observed with an inverted confocal microscope (FV1000, Olympus) with a water-immersion $40 \times$ objective lens (LUMPlanFl NA0.8, Olympus) with Immersol W (Carl Zeiss) immersion oil. We selected the region in which there was a single EGFP/ $\mathrm{mKO} 2-\mathrm{F}$-expressing cell with complete radial glia morphology. The picture was scanned at $256 \times 512$ pixels with $8-18 \mathrm{Z}$ sections $(2-4$ $\mu \mathrm{m}$ intervals), and pictures were taken at $4-20 \mathrm{~min}$ intervals. The draft of samples was adjusted by hand every $6-12 \mathrm{~h}$. The time-lapse data were assembled by Fluoview software (Olympus), and stacked three-dimensional (3D) images were constructed by Volocity 5.2 software (Improvision).

Immunohistochemistry. Brains were fixed in $0.5 \%$ paraformaldehyde in phosphate buffer, $\mathrm{pH} 7.4$, at room temperature for $20 \mathrm{~min}$, followed by cryoprotection in $20 \%$ sucrose overnight at $4^{\circ} \mathrm{C}$, and embedded in OCT compound (Sakura Tissue-Tek). Cryostat sections $(10 \mu \mathrm{m})$ were treated with blocking buffer ( $10 \%$ donkey serum and $0.1 \%$ Tween 20 in TBS, pH 7.4) for $30 \mathrm{~min}$ at room temperature, followed by incubation with primary antibodies diluted in the same buffer overnight at $4^{\circ} \mathrm{C}$. Sections were washed three times in $0.1 \%$ Tween 20 in TBS (TBST). Hes 1 was detected with a tyramide signal amplification system (PerkinElmer). To visualize the basal process, we performed vibratome section staining, as shown in Figure 4 . The $4 \%$ low melting point agar (ultrapure, Invitrogen)-embedded fixed brains were microdissected to $200 \mu \mathrm{m}$ with a vibratome (VT1000S, Leica). The vibratome slices were treated with blocking buffer overnight at $4^{\circ} \mathrm{C}(10 \%$ donkey serum and $0.1 \%$ Triton $\mathrm{X}-100$ in TBS, $\mathrm{pH} 7.4$ ), followed by incubation with primary antibodies diluted in the same buffer for $2 \mathrm{~d}$ at $4^{\circ} \mathrm{C}$. The sections were washed three times in TBST for $1 \mathrm{~h}$ and incubated overnight at $4^{\circ} \mathrm{C}$ with the secondary antibodies. The sections were washed two times in TBST for $1 \mathrm{~h}$ and then overnight at $4^{\circ} \mathrm{C}$. After being washed, the sections were sandwiched between two cover glasses with mounting medium (Prolong Gold, Invitrogen) and small cover glasses to prevent crushing. Specimens were then analyzed with a confocal microscope (FV1000, Olympus). The same procedure was used for staining of cultured slices following time-lapse imaging. The following primary antibodies were used: rabbit polyclonal anti-Pax6 (1:500; AB5409, Millipore), rabbit polyclonal anti-Ki67 (1: 500; NCL-Ki67p, Nococastra), rat monoclonal anti-Hes1 (1:1000; clone NM1, MBL), chicken polyclonal anti-EGFP (1:1000; GFP-1020, Aves Labs), rabbit polyclonal anti-Tbr2 (1:500; AB9618, Millipore), and mouse monoclonal anti-Phospho (Ser55) vimentin (1:250; clone 4A4, MBL). Secondary antibodies were conjugates of HRP, Cy2, Cy3, or Cy5 (1:1000, Jackson ImmunoReseach).

Statistical analysis. The quantified data were evaluated by Student's $t$ test using Prism 4 software (Graph Pad Software) except for Figure 6. The data in Fig. 6 were evaluated by one-way ANOVA with a post-Bonferroni multiple-comparison test using Prism4 software.

\section{Results}

Daughter cell fate of apical divisions is independent of the size of the inherited apical membrane

We first investigated the relationship between the inheritance of the apical junction and daughter cell fate by viewing brain slices from the apical side. This was accomplished using in utero electroporation to introduce two types of plasmids that were used in a previous study (Konno et al., 2008) into the developing mouse neocortex: ZO1-EGFP (an EGFP fusion of an apical junction component) and a centrosomal marker, PACT-mKO1 (the PACT domain of pericentrin-2 fused with $\mathrm{mKO} 1)$. This assay enabled us to distinguish the daughter cell 
fate of the two cell types. One type is the radial glia cells, which maintain the apical junction during interkinetic nuclear migration until the next division; these cells are hereafter termed "apical progenitors" to emphasize that they divide at the apical surface after interkinetic nuclear migration. The other type is the nonradial glial cells, including neurons, outer ventricular zone (VZ) progenitors (see Fig. 4), and "basal progenitors," which are intermediate progenitors that divide once in the subventricular zone (SVZ) into a pair of neurons. Those cells lose the apical junctions either during division or by committing to differentiation, and leave the VZ.

Twenty-four hours after electroporation at E12.5, we initiated long-term observations for $2 \mathrm{~d}$, corresponding to the midneurogenesis stage (E13.5-E15.5) when apical progenitor cells extensively produce neurons. At the majority of the apical progenitor divisions, the apical junctions are split into two daughters under our experimental conditions, as previously described (Konno et al., 2008). Two centrosomes that have partitioned into the two daughter cells return to the apical surface shortly after cleavage (Fig. $1 A, B$ ). When the daughter cell assumes the apical progenitor fate (i.e., maintaining radial glial morphology), the centrosome remains at the apical surface throughout the cell cycle until the next mitosis. In contrast, the centrosome in differentiating cells (basal progenitors or neurons) detaches from the apical surface and migrates, along with the cell body, away from the VZ surface (Wang et al., 2009). More than half of those pairs adopted asymmetric cell fates (Fig. $1 B$ ). We never observed that the daughter cells of apical divisions that had once lost their apical junctions reestablished the apical attachment by re-extension of the apical process during the period from E13.5 to 15.5.

We examined whether there was a correlation between the daughter cell fate of apical divisions and the size of the apical domain (inner region of the ZO1 ring) inherited by a daughter cell. There was no significant difference in the inherited apical area between the apical progenitor and those cells that retract the apical process after division (Fig. 1C). Additionally, we failed to find a correlation between the cell fate of the two daughter cells and the ratio of their apical membrane size at cleavage (Fig. $1 D$ ). We also investigated the inheritance of the apical junction using PAR3-EGFP instead of ZO1-EGFP and obtained essentially the same results (supplemental Fig. S1, available at www.jneurosci. org as supplemental material). These results strongly suggest that cell fate decision of self-renewal versus differentiation does not depend on the inheritance of the apical junctions or the inherited apical domain size.

\section{Self-renewing progenitors are generated from the daughter cells that inherit the basal process}

We next investigated the correlation between the inheritance of the basal process and the daughter cell fate of apical progenitors in the cross-sectional view of the brain slices. We clonally labeled apical progenitors with a Cre-loxP clonal expression plasmid system (Morin et al., 2007; Kato et al., 2010) to monitor the entire radial glial morphology at the single-cell level. In addition, to clarify which daughter cell of the apical division inherited the thin radial process, we labeled the cytoplasm with EGFP and the cell membrane with mKO2-F, which contained a CAAX farnesylation motif that localizes to the membrane. Electroporation was performed at E13.5, and $24 \mathrm{~h}$ later we began culturing the brain slices and observed them for $2 \mathrm{~d}$ (corresponding to E14.5-E16.5). Under our experimental conditions, all apical progenitors divided asymmetrically with regard to the partition of the basal process: one daughter cell inherited a long basal process, and the other daughter cell did not inherit any basal processes (Fig. 2A). We further followed the fate of these daughter cells through one cell cycle by monitoring cell morphology, the mode of nuclear migration (INM or a simple apical-to-basal migration), and mitotic ability and position (Fig. $2 \mathrm{~B}$ ). Apical progenitors displayed radial glial morphology, underwent INM, and divided at the apical surface. Nonsurface progenitors did not undergo INM and divided at the SVZ. This class of progenitors included the basal progenitors that divided only once into a pair of neurons, and also outer VZ progenitors (see Fig. 4), although our time-lapse observations during one cell cycle were unable to distinguish between the two types of progenitors. Daughter cells that did not divide for $>35 \mathrm{~h}$ were regarded as postmitotic cells (neurons). We found that the daughter cells that inherited the basal process maintained proliferative activity and assumed the fate of either an apical progenitor or nonsurface progenitor (Fig. 2B). Conversely, those that did not inherit the basal process never established it and committed to the fate of either a nonsurface progenitor or a neuron (Fig. $2 B$ ). These observations are also consistent with those from a previous study (Noctor et al., 2001).

We also examined the expression of Hes1 and Tbr2 to determine whether Notch is inactivated or not (resulting in differentiation) in the daughter cells of apical divisions in the slice cultures (Fig. 2C,D). Hes1 was expressed in $66 \%$ of the daughter cells that inherited the basal process, while $15.7 \%$ of them expressed Tbr2. In contrast, 10.8 and $56.8 \%$ of those that did not inherit the basal process expressed Hes1 and Tbr2, respectively. Consistent with the results of time-lapse imaging, these data indicate that Notch is highly activated in the daughter cells that inherit the basal process and that Notch tends to be inactivated in those that do not inherit the basal process. Together, our results suggest that during the mid-neurogenic stage, the daughter cells of apical divisions can self-renew as apical progenitors only when they inherit both apical and basal processes.

\section{Notch activation does not affect the division pattern but induces the basal process}

Using slice cultures, our observations indicate that the majority of apical progenitors undergo asymmetric divisions that generate one daughter cell that inherits the complete epithelial structure (with a radial glial morphology) and another that inherits the apical membrane but not the basal process. Self-renewal of neural progenitors requires Notch signaling, which activates the expression of the Hes transcription factors. In turn, they repress the expression of proneural genes that promote neuronal commitment (Yoon and Gaiano, 2005). The proportion of self-renewing progenitors (radial glia) in the developing dorsal cortex is increased by Notch activation via overexpression of the NICD (Gaiano et al., 2000). By overexpressing NICD, we investigated whether forced Notch activation affects the typical division pattern of the apical progenitors and found that all NICD-expressing progenitors segregate the basal process into one daughter cell, as observed in the control brain slices (Fig. 2). This indicates that constitutive Notch activation does not affect the asymmetric segregation of the basal process during apical divisions (Fig. $3 A$ ). Instead, we observed that the daughter cell that did not inherit the basal process re-established the basal process when the nucleus was migrating from the apical surface. These cells extended the basal process to $\sim 70 \%$ of the length $(254 \pm 17.7 \mu \mathrm{m}, n=13)$ of the fully extended radial process ( $354 \pm 12.2 \mu \mathrm{m}, n=10$ ) during the first round of cell cycle, and INM continued to extend it, regenerating the radial morphology (Fig. $3 A$ ). Self-renewing progenitors undergoing INM along the radial process were produced at the same rate from both types of daughter cells, regardless of 
A

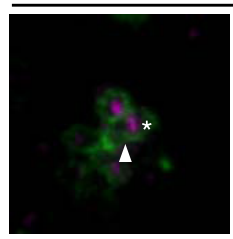

Oh:00m

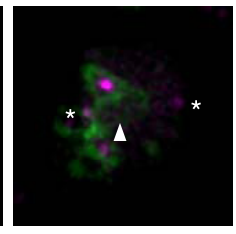

$0: 37$
First cell division

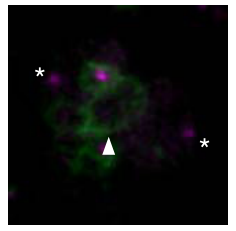

$0: 45$

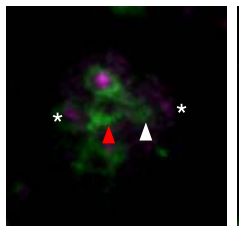

$1: 06$

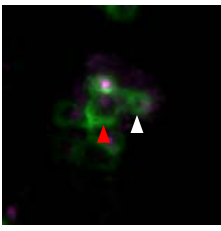

2:06

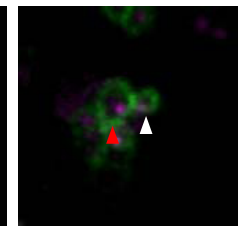

$4: 58$
Exit from ventricular surface

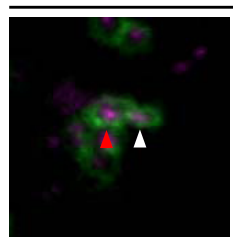

$8: 40$

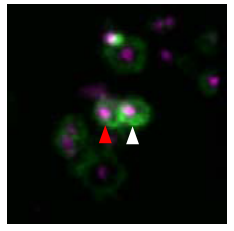

$16: 27$

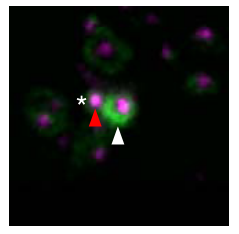

20:26

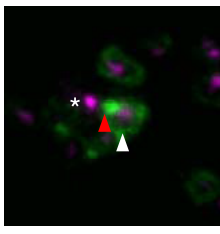

24:26

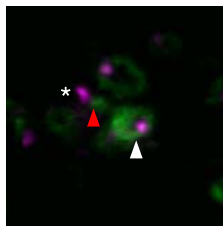

$26: 21$

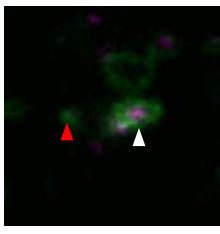

$28: 12$
Second cell division

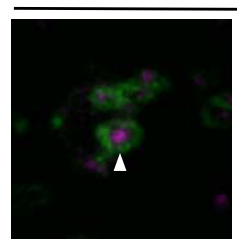

$31: 05$

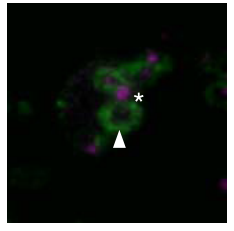

$31: 17$

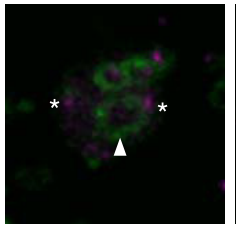

$31: 38$

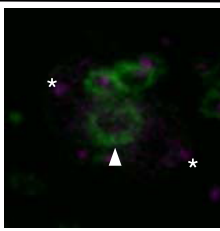

$31: 54$

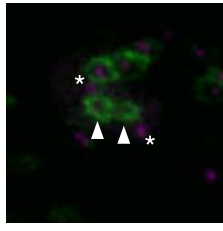

$32: 15$

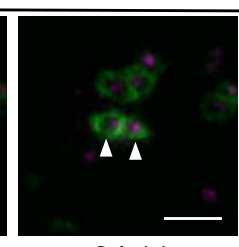

$34: 11$
B

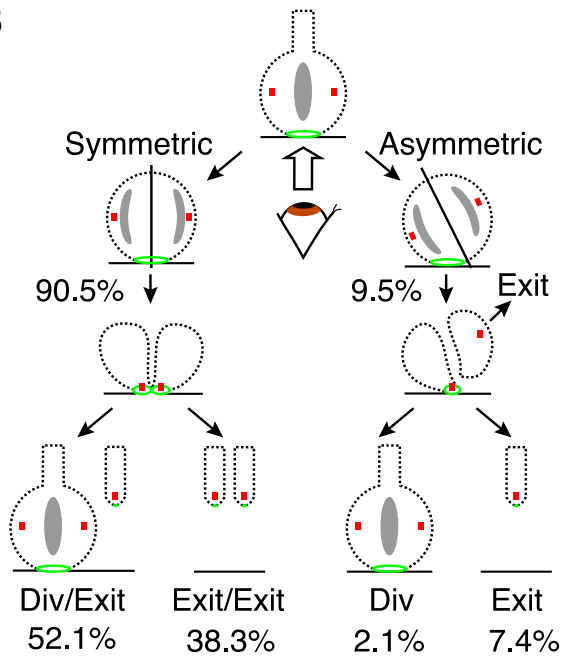

C

Inherited apical domain size $\left(\mu \mathrm{m}^{2}\right)$
D Apical domain size between a pair of cells $\left(\mu \mathrm{m}^{2}\right)$
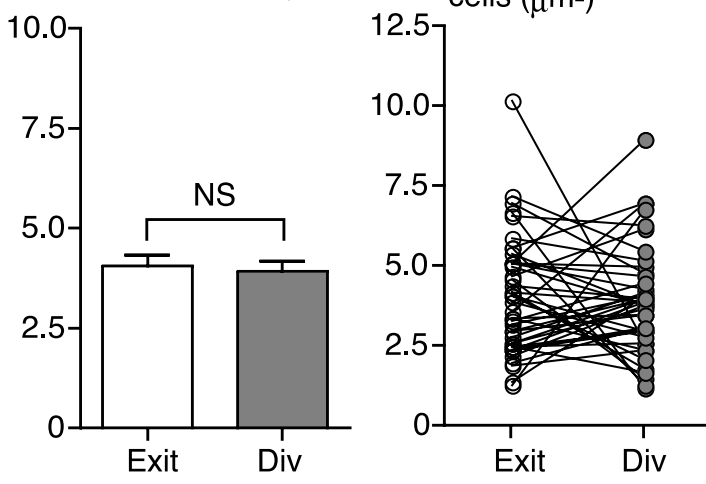

Figure 1. Time-lapse analysis of apical junction inheritance and daughter cell fate. $A$, Time-lapse movie of the asymmetric cell division of the apical progenitor observed from the apical surface. The brains were electroporated with the plasmids for EGFP-Z01 $(0.2 \mu \mathrm{g} / \mu \mathrm{l})$ and PACT-mK01 $(0.2 \mu \mathrm{g} / \mu \mathrm{l})$ at E12.5, and time-lapse imaging was performed $24 \mathrm{~h}$ later. The daughter cell fate was followed by EGFP-Z01 (green ring) and PACT-mK01 (magenta dots) for $2 \mathrm{~d}$. Top panels, The first cell division of the apical progenitor. At time 0, the duplicated centrosomes (asterisks) are located at the apical surface. They migrate into the middle of the cell body and are laterally segregated during mitosis. At the end of mitosis, the apical junction divides symmetrically into both daughter cells (white and red arrowheads). Middle panels, The subsequent retraction of the apical end foot. One of the daughter cells (red arrowhead) shrinks the apical junction and detaches from the apical surface together with the centrosome (red arrowhead and asterisk). Bottom panels, The second cell division of the sibling daughter cell. This cell (white arrowhead) maintains the apical junction and divides again at the apical surface. The apical junction is also symmetrically divided into both daughter cells. Scale bar, $5 \mu \mathrm{m}$. Times are given as hours:minutes. $\boldsymbol{B}$, Schematic drawing of the partition of the apical junction during apical progenitor cell division and the behavior of daughter cells for the two-cell cycle periods. A total of 94 apical divisions were followed. The numbers indicate the percentage of events that are described as follows: "Symmetric" shows symmetric division of the apical junction into both daughter cells; "Asymmetric" shows asymmetric division of the apical junction into two daughter cells, one of which loses the apical junction and disappears from the ventricular zone; "Div" shows the daughter cell dividing again at the apical surface, indicating the apical progenitor fate; and "Exit" shows the cell retraction of the apical endfoot with the centrosome and exit from the ventricular zone, indicating neural differentiation. $\boldsymbol{C}$, The inherited size of the apical junction is not significantly different between the apical progenitor fate (Div) and the differentiated cell fate (Exit). The size of the inner region of the EGFP-Z01 ring was measured just after cytokinesis of the first apical division of the asymmetric cell pair fate as shown in $\boldsymbol{B}$ (Div/Exit pair, $n=49$ ). Error bar represents SEM. NS, Not significant. Student's $t$ test, $p=0.69$. $\boldsymbol{D}$, The ratio of inherited size of the apical junction between the pair of daughter cells shown in $\boldsymbol{B}$ (Div/Exit pair, $n=49$ ). There is no correlation between sibling cell fates and inherited apical domain size.

whether they had inherited a basal process (Fig. 3B). This is most likely to cause an increase in the proportion of the apical progenitors, as observed in a previous report (Gaiano et al., 2000). This observation indicates a strong correlation between progenitor self-renewal and the basal process.
Self-renewing progenitors outside of the VZ retain the basal process but not the apical process

We next investigated the relationship between epithelial substructures and the self-renewal of apical progenitors. We were previously able to segregate the apical domain and basal domain 


\section{A E14 Apical progenitor/Nonsurface progenitor}

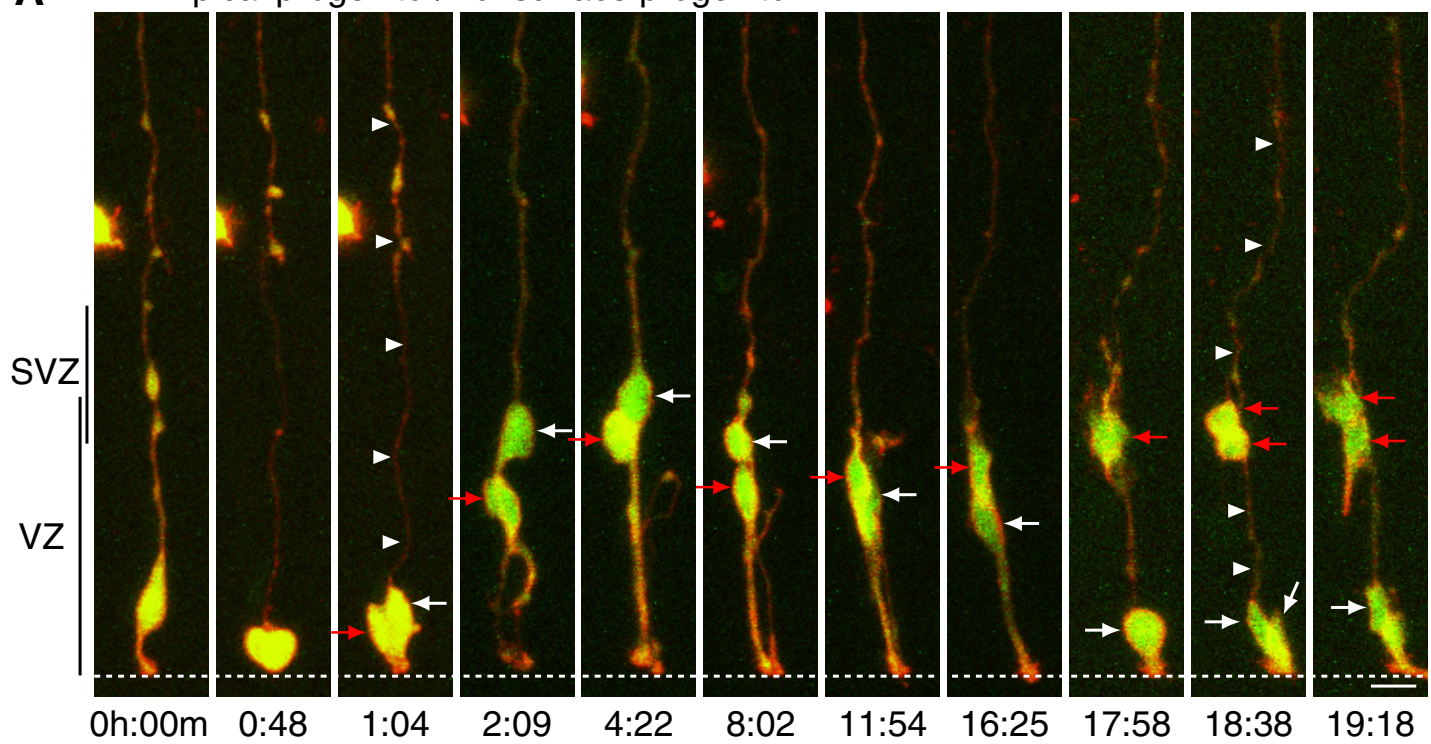

B Basal process Daughter cell fate (\%) inheritance

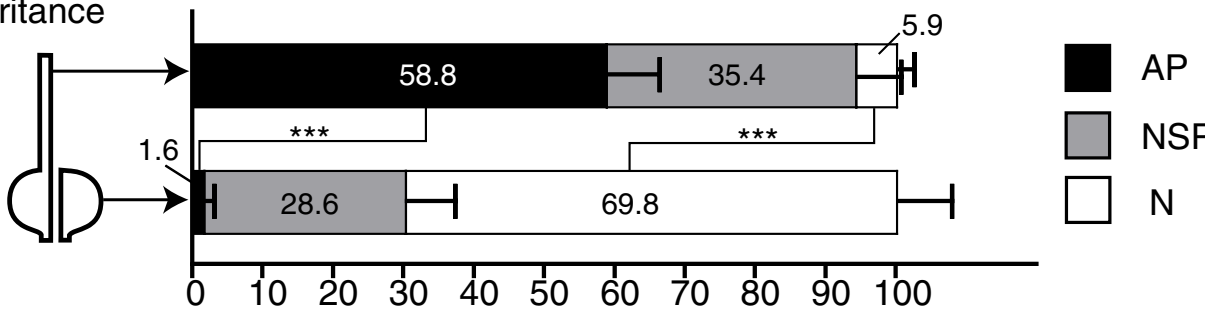

C

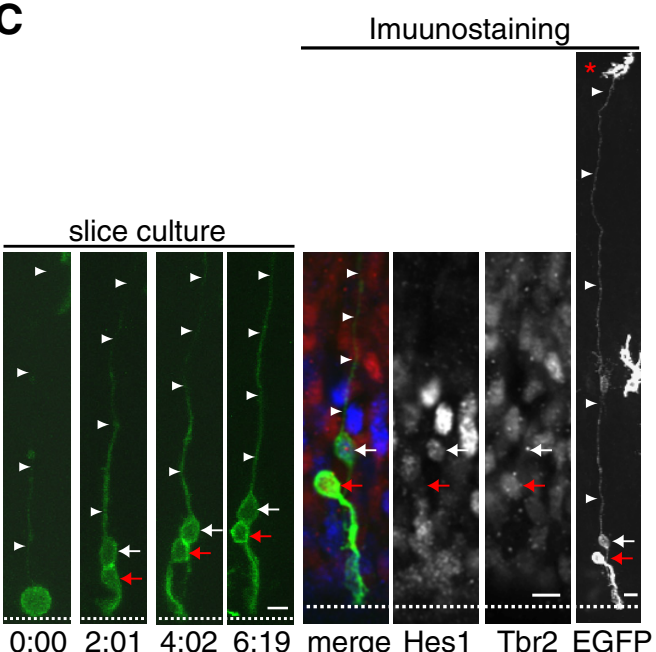

D

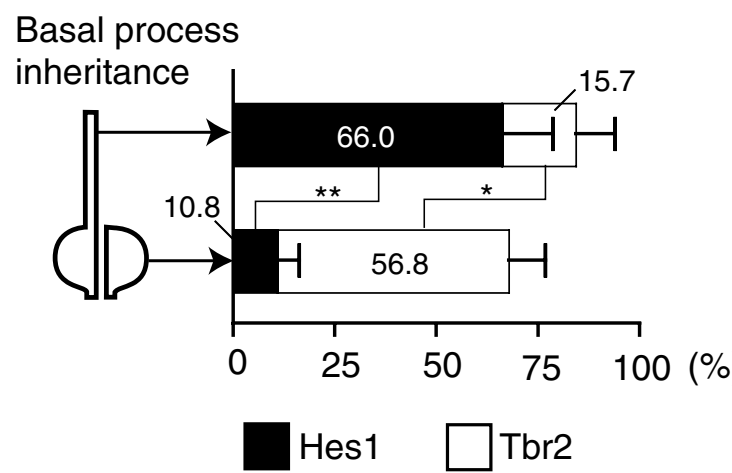

Figure 2. The apical progenitor inherits the entire epithelial structure. $A$, Time-lapse movie of the asymmetric cell division of the apical progenitor. The brains were electroporated with the plasmids for Cre, FloxP-EGFP, and-mK02-FatE13.5, and time-lapse imaging was performed $24 \mathrm{~h}$ later. The daughter cell fate and morphology were observed for $2 \mathrm{~d}$. The apical progenitor divides at the apical surface (0:48 -1:04). One of the daughter cells (white arrow) inherits the basal process (white arrowhead), undergoes INM (1:04-17:58), and divides again at the apical surface (17:58 -18:38). The basal process is also asymmetrically divided at the second cell division (white arrowhead, 18:38). The sibling daughter cell (red arrow) does not inherit the basal process. The cell migrates to the basal side and loses the apical attachment. Finally, the cell divides at the basal side(18:38-19:18). The apical surface is down (dashed lines). Scale bar, $10 \mu \mathrm{m}$. Times are given as hours:minutes. $\boldsymbol{B}, \mathbf{Q}$ uantified results of the time-lapse analysis of daughter cell fate. Most of the apical progenitors are derived from the daughter cells inheriting the basal process. The upper bar shows the fate of the cells that inherited the basal process (AP, $58 \pm 7.7 \%$; NSP, $35 \pm 6.0 \% ; \mathrm{N}, 5.9 \pm 2.4 \%$; $n=73$ pairs from eight experiments with three brains for each experiment), and the lower bar shows the fate of the cells that did not inherit the basal process (AP, $1.6 \pm 1.6 \% ; \mathrm{NSP}, 28.6 \pm 7.2 \% ; \mathrm{N}, 69.8 \pm$ 8.0\%). Error bar represents SEM. Student's t test, ${ }^{* * *} p<0.0001$ for both AP and N. AP, Apical progenitor; NSP, non-surface progenitor; N, neuron (postmitotic cell). The definition of the cell fate is described in the results. C, The fate of the daughter cells of apical divisions in the slice culture. The brains were electroporated with the plasmids for Cre, FloxP-EGFP-F, and-LGN-CatE13.5, and time-lapse imaging started 24h later. The slice was fixed 2-16 h after the first apical division of a radial glial cell in the slice culture and stained for Hes 1 and Tbr2. The right panel indicates that the monitored radial glial cell extended the radial fiber from the ventricular surface to the pial surface to form the basal endfoot (red asterisk). See $\boldsymbol{A}$ for symbols. Scale bar, $10 \mu \mathrm{m}$. $\boldsymbol{D}, \mathbf{Q}$ uantification of Hes 1 -positive cells (Hes 1 ) and Tbr2-positive cells (Tbr2) in the daughter cells of apical divisions in the slice culture. The upper bar indicates the daughter cells that inherited the basal process (Hes1, 66.02 $\pm 12.6 \%$; Tbr2, 18.2 $\pm 9.7 \% ; n=37$ pairs from three brains); the lower bar, the daughter cells that did not inherited the basal process (Hes1, $10.8 \pm 5.5 \%$; Tbr2, $56.8 \pm 9.2 \%$ ). Error bars represent SEM. Student's $t$ test, ${ }^{* *} p=0.016$ for Hes 1 and ${ }^{*} p=0.04$ for Tbr2. 


\section{A E14 NICD overexpression}

\section{Apical progenitor/Apical progenitor}
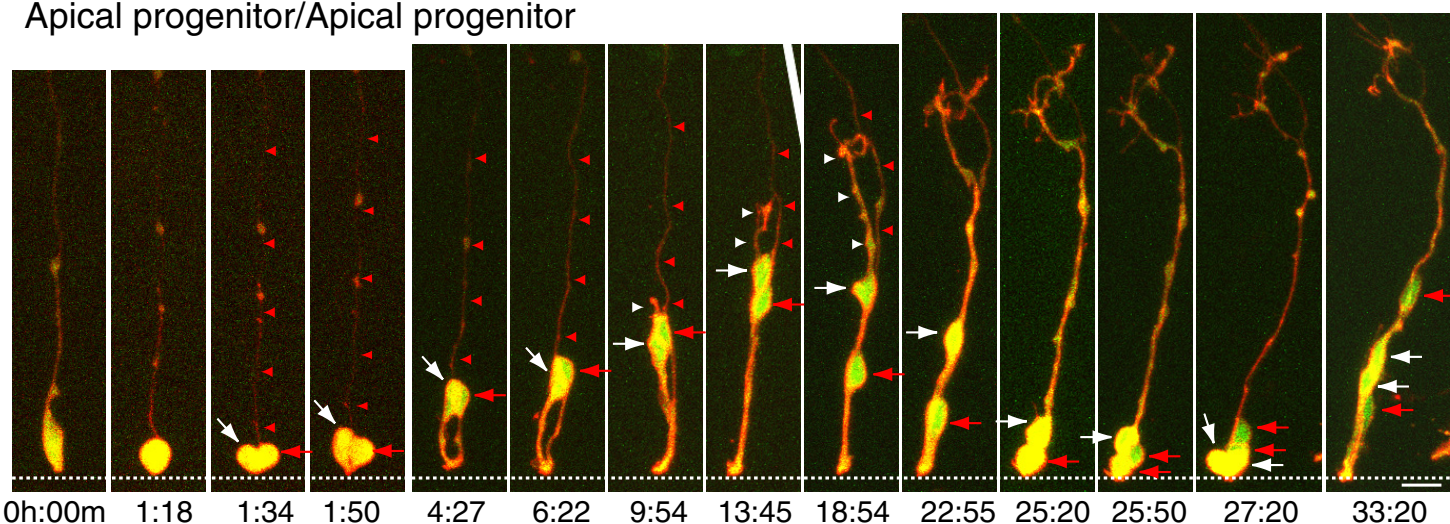

\section{B Basal process} inheritance

\section{Daughter cell fate (\%)}

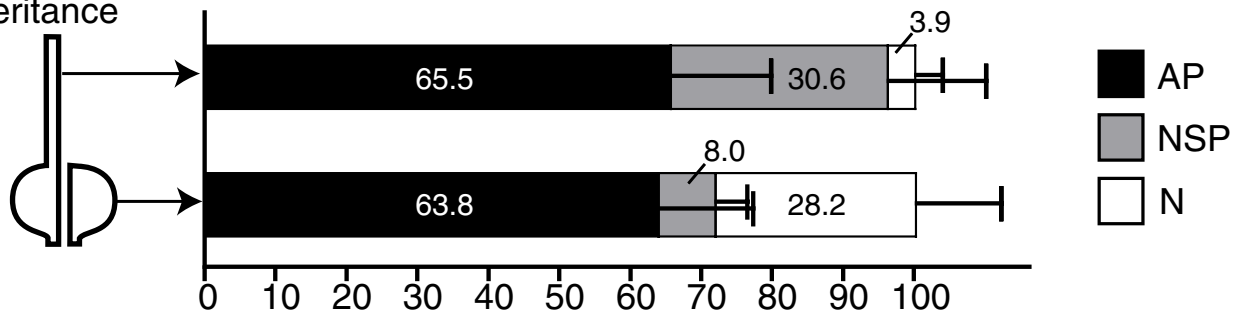

Figure 3. Notch activation promotes the regeneration of the basal process without affecting the dividing pattern of the basal process during the apical progenitor cell division. $A$, Time-lapse movie of the regeneration of the basal process after apical progenitor cell division. The brains were electroporated with the plasmids for Cre, FloxP-EGFP,-mKO2-F, and -NICD atE13.5, and time-lapse imaging was performed $24 \mathrm{~h}$ later. The daughter cell fate and morphology were observed for $2 \mathrm{~d}$. The basal process is asymmetrically distributed into one of the daughter cells (red arrow and red arrowhead) but not the other (white arrow). The daughter cell that inherited the basal process (red arrow) undergoes interkinetic nuclear migration (4:27-22:55) and then divides at the apical surface (25:20-25:50), whereas another daughter cell starts to establish the basal process (white arrowhead, 9:54) and then establishes the radial glial morphology. Finally, the cell divides at the apical surface (27:20). The apical surface is down (dashed lines). The radial process growing from the daughter that did not inherit it reached the length of $320 \mu \mathrm{m}$, which was $90 \%$ of the width of the cortex. Scale bar, $10 \mu \mathrm{m}$. Times are given as hours:minutes. $\boldsymbol{B}$, Quantified results of the time-lapse analysis of daughter cell fate. Both daughter cells acquired the apical progenitor fate at a similar rate, independent of the inheritance of the basal process. The upper bar shows the fate of the cells that inherited the basal process (AP, $65.5 \pm 14.3 \%$; NSP, $30.6 \pm 13.8 \%$; and N, $3.9 \pm 3.9 \%$; $n=29$ pairs from four experiments with three brains each), and the lower bar shows the fate of the cells that did not inherit the basal process (AP, $3.8 \pm 13.4 \%$; NSP, $8.0 \pm 4.6 \%$; and N, $28.2 \pm$ 12.0\%). Error bars represent SEM. Student's $t$ test, $p=0.93$ for AP, 0.17 for NSP and 0.10 for N. AP, Apical progenitor; NSP, no-surface progenitor; and N, neuron (postmitotic cell).

of dividing apical progenitors into separate daughter cells by perturbing the mitotic orientation (Morin et al., 2007; Konno et al., 2008). We found that the more basal daughters from oblique or vertical divisions, which presumably inherit only the basal processes, migrate out of the VZ to become either self-renewing progenitors or non-self-renewable basal progenitors, while the more apical daughters, which inherit only the apical processes, commit to the differentiating fate. We took advantage of this characteristic as a strategy to question the roles of the apical and basal domains of the radial process in cell fate determination. We compromised the function of LGN, which is necessary for correct orientation of the cleavage plane (Morin et al., 2007; Konno et al., 2008), by expressing the dominant-negative form of the LGN-C (Morin et al., 2007) (supplemental Fig. S2, available at www. jneurosci.org as supplemental material). We then monitored the cell morphology and fate of individual daughters from normal and oblique apical divisions in the long-term brain slice cultures for two more cell cycles (within a 3-cell cycle period). In the normal brain, the majority of apical divisions showed a cleavage plane that was nearly vertical to the epithelial plane. However, when LGN-C was expressed under clonal conditions, the apical divisions frequently became oblique (supplemental Fig. S2, available at www.jneurosci.org as supplemental material), resulting in the cleavage plane increasingly bypassing the apical surface (Konno et al., 2008). Consequently, we observed that the daugh- ter cells that inherited the basal process divided outside of the $\mathrm{VZ}$ more often than in the normal brain slices $(71.1 \pm 6.8 \%$ vs $35.4 \pm$ $6.0 \%$ ) (compare Figs. $4 B, 2 B$ ). The daughters lacking the basal process differentiated into neurons $(75.4 \pm 5.4 \%$ ) (Fig. $4 B$ ) or basal progenitors $(24.7 \pm 5.4 \%$ ) (Fig. $4 B$ ), similar to the control slices (Figs. $2 B, 4 B$ ).

The two daughter cells from oblique apical divisions began to migrate basally while associating with each other via a thin process (Fig. 4A). The apical daughter cell that lacked the long basal process initially retained the apical endfoot but subsequently lost it (by commitment to differentiation). In contrast, the more basal daughter cells inherited the basal processes (but apparently not the apical endfoot) and migrated ahead of the more apical daughter cells to exit the VZ. These cells did not return to the apical surface or divide asymmetrically to segregate the basal process into one daughter cell. We found that these basal daughter cells divided twice (Fig. 4A, 21 h: 39 min-22 h: 00 min and 41 h: 18-38 $\mathrm{min}$ ) outside of the $\mathrm{VZ}$ at a high frequency $(43.7 \pm 6.3 \%)$ (Fig. $4 B$ ) during the period of slice culture. At these sequential divisions outside of the $\mathrm{VZ}$, the basal process was always segregated into the mitotic daughter in the next cell cycle, indicating the perfect correlation of self-renewability with the inheritance of the basal process. In contrast, the sibling daughter cells that did not inherit the basal process became postmitotic in our observations (Fig. 4A). These postmitotic daughter cells maintained contact 
A E14 LGN-C overexpression Outer VZ progenitor/Neuron (post-mitotic)

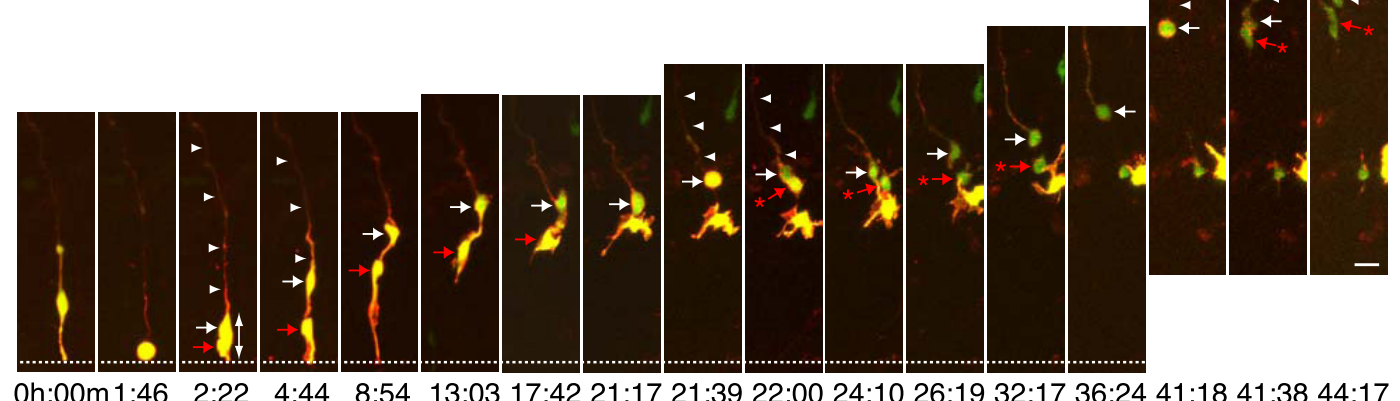

B

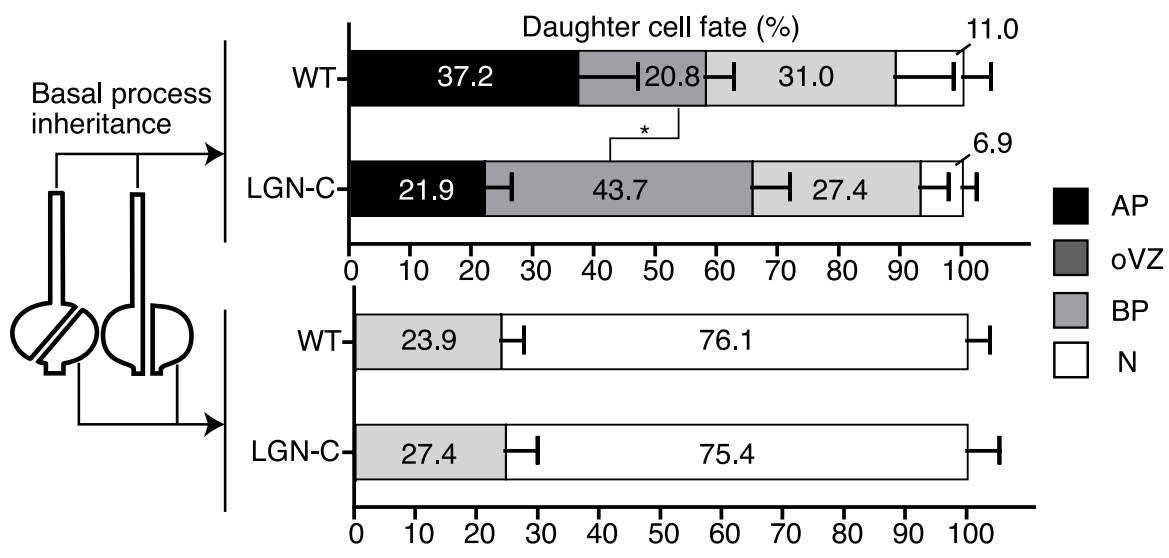

C

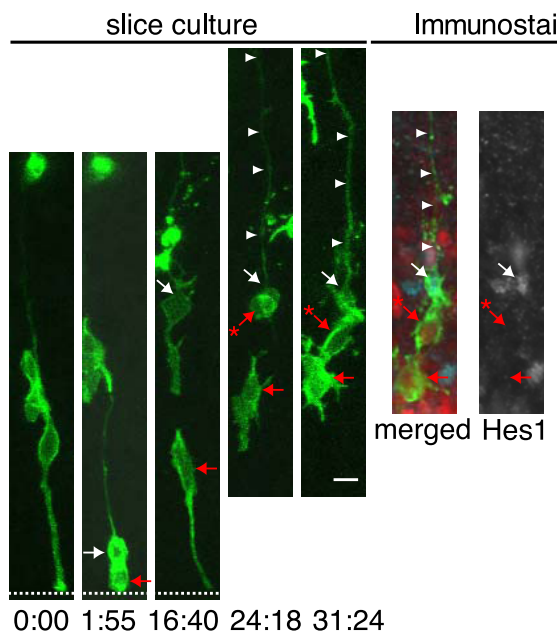

D The marker expression of daughter cells at the first division of the oVZ progenitor

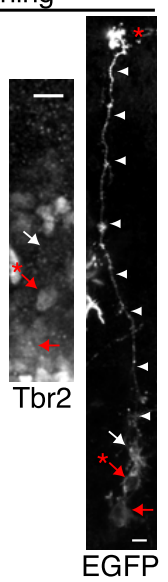

Basal process

inheritance

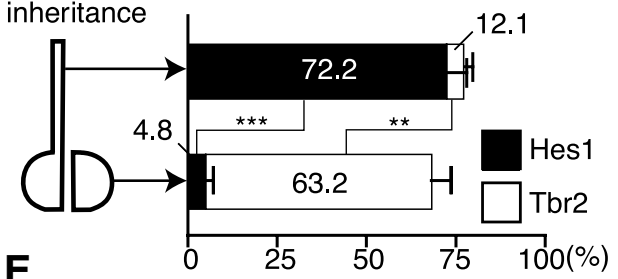

E

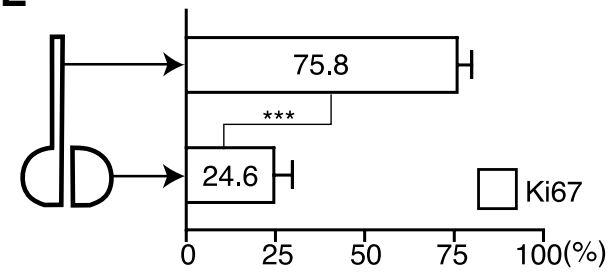

Figure 4. Spindle misorientation induces the self-renewing progenitor cells that inherit only the basal process outside of the VZ.A, Time-lapse movie of the self-renewing progenitor cell outside of the VZ that was induced by spindle misorientation. The brains were electroporated with the plasmids for Cre, FloxP-EGFP,-mK02-F, and -LGN-CatE13.5, and time-lapse imaging was performed $24 \mathrm{~h}$ later. The daughter cell fate and cell morphology were observed for $3 \mathrm{~d}$. The apical progenitor divides nearly vertically (2:22), and the epithelial structure is split by cytokinesis. The upper daughter cell (white arrow) inherits and maintains the basal process (white arrowhead). The cell divides two times (21:39-22:00 and 41:18 - 41:38) outside of the VZ and produces the daughter cell without the basal process (red arrow with asterisk), whereas the lower cell (red arrow) does not inherit the basal process. The cell assumes a multipolar cell morphology and stops dividing. The apical surface is down (dashed lines). Scale bar, $10 \mu \mathrm{m}$. Times are given as hours:minutes. $B$, Quantified results of the time-lapse analysis of daughter cell fate in the wild-type and the LGN-C overexpressing conditions. The upper bars show the fate of the cell that inherited the basal process in the wild-type (AP, $37.2 \pm 9.9 \% ; 0 \mathrm{VZ}, 20.8 \pm 4.8 \% ; \mathrm{BP}, 31.0 \pm 9.6 \%$; and N, $11.0 \pm 4.7 \% ; n=37$ pairs from four experiments with three brains each) and in the $\mathrm{LGN}$-Coverexpressing condition (AP, $21.9 \pm 4.6 \% ; 0 \mathrm{VZ}, 43.7 .8 \pm 6.3 \% ; \mathrm{BP}, 27.4 \pm 4.8 \% ;$ and N, $6.9 \pm 2.4 \% ; n=41$ pairs from four experiments with three brains each), and the lower bars show the fate of the cell that did not inherit the basal process in the wild-type (AP, $0 \% ; 0 \mathrm{VZ}, 0 \% ; \mathrm{BP}, 23.9 \pm 3.9 \%$; and N, $76.1 \pm 3.9 \%$ ), and on the LGN-Coverexpressing condition (AP, $0 \% ; 0 \mathrm{VZ}, 0 \%, \mathrm{BP} 24.7 \pm 5.4 \% ; \mathrm{N}, 75.4 \pm 5.4 \%)$. Error bars represent SEM. Student's t test, ${ }^{*} p=0.027$ for oVZ. AP, Apical progenitor; $0 \mathrm{VZ}$, outer VZ progenitor; $\mathrm{BP}$, basal progenitor; $\mathrm{N}$, neuron (postmitotic cell). The definition of the cell fate is described in the results. C, The fate of the daughter cells of an outer VZ division in slice culture expressing LGN-C. The daughter cells of an oblique apical division were monitored in the slice culture (labeled as described in Fig. 20) for 2-16 h after the first outerVZdivision, followed by fixation for staining for Hes 1 and Tbr2. Symbols areindicated in A. Right, The basal process of the Hes1-positive daughter reaches the pia to form the basal endfoot (red asterisk). Scale bar, $10 \mu \mathrm{m}$. D, Quantification of Hes1-positive cells and Tbr2-positive cells in the daughter cells of outerVZdivisions on overexpression of LGN-C. The upper bar indicates the daughter cells that inherited the basal process (Hes 172.2 $\pm 5.5 \%, \mathrm{Tbr} 212.1 \pm 6.7 \% ; n=36$ pairs from three brains); the lower bar shows the daughter cells that did not inherited the basal process (Hes $14.8 \pm 2.4 \%$, Tbr2 63.2 $\pm 5.6 \%$ ). Error bars represent SEM. Student's $t$ test, ${ }^{* * *} p=0.0004$ for Hes1; ${ }^{* *} p=0.043$ for Tbr2. E, Quantification of the Ki67-positive daughter cells of outer VZ divisions in the slice cultures expressing LGN-C. Above is the percentage of Ki67-positive cells in the daughter cells that inherited the basal process $(75.8 \pm 4.1 \% ; n=33$ pairs from three brains); below is the percentage of Ki67-positive daughter cells that did not inherited the basal process $(24.6 \pm 5.2 \%)$. Error bars represent SEM. Student's $t$ test, ${ }^{* * *} p=0.0015$. 
with the cell body of the self-renewing sister cell for several hours, while the self-renewing sister cell gradually migrated to a more basal position until the next cell division. These data indicate that spindle misorientation at apical divisions induces self-renewing progenitors outside of the VZ, as predicted in our previous study (Konno et al., 2008; Shioi et al., 2009), and these progenitors retain the basal but not the apical process, generating neurons by asymmetric divisions. Hereafter, we call these progenitors "outer VZ progenitors" to distinguish them from the non-self-renewable basal progenitors (which divide once into a pair of neurons) and the apical progenitors that undergo interkinetic nuclear migration in the VZ to self-renew.

To confirm the results obtained by this cell lineage analysis, we examined Ki67 expression as a proliferation marker, Hes1 expression as a marker of self-renewal, and Tbr2 expression as a marker of differentiation in the daughter cells of the first ectopic division (outside of the VZ) in cultured slices expressing LGN-C (Fig. 4C-E). Hes1 was expressed in $72.2 \%$ of daughter cells that inherited the basal process at the ectopic division, while $12.1 \%$ of them expressed Tbr2. In contrast, Hes 1 and Tbr2 were expressed in 14.8 and $63.2 \%$ of those that did not inherit the basal process, respectively. Similar results were obtained with Ki67 expression. These data indicate that Notch is highly activated in daughter cells that inherit the basal process in ectopic divisions while Notch tends to be inactivated in those that do not inherit the basal process. Thus, lineage analysis of oblique divisions and gene expressions of their daughter cells consistently indicate that the inheritance of the basal process is highly correlated with the acquisition of self-renewal.

\section{Outer VZ progenitors retaining the basal process exist in the normal developing mouse brain}

Our previous study showed that even in normal brain slices, there is a low frequency of apical divisions that segregate the apical membrane into only one daughter cell due to oblique spindle orientation (Konno et al., 2008). We confirmed that this type of apical division occurs throughout the mid-neurogenic stage, with a slight increase in our time-lapse imaging condition $[16.6 \%$ on average: $13.7 \%$ at E13.5 $(n=234), 16.0 \%$ at E14.5 $(n=131)$, and $20.0 \%$ at E16.5 $(n=80)]$, suggesting that a small number of self-renewing outer $\mathrm{VZ}$ progenitors with basal processes are formed from apical divisions, even in the normal developing brain. We tested this possibility using time-lapse imaging of the normal brain slices without electroporation with LGN-C. In these normal brain slices, we observed that at a low frequency of apical divisions one daughter cell was generated that retained the apical process and typically differentiated, and another daughter cell retained the basal process and typically exited the VZ, continuing self-renewal outside of the VZ in the normal brain slice by low-frequency oblique divisions where the cleavage plane bypasses the apical membrane.

We next investigated whether outer VZ progenitors retain the basal process in vivo. To clearly visualize the morphological features of individual cells, including the basal processes in fixed sections, EGFP was expressed at low cell densities in the developing brain via a Cre-loxP expression plasmid system (Fig. 5). Two days after electroporation at E13.5, $5.6 \pm 0.6 \%$ and $11.8 \pm 1.9 \%$ of EGFP-expressing cells retained the basal process without the apical process in the control and LGN-C-expressing brain sections, respectively (Fig. 5C). These EGFP-expressing cells were mainly distributed outside the VZ (Fig. 5E, SVZ and intermediate zone). In the EGFP-positive cells with the basal processes, we examined the expression of the markers of the apical progenitors, Pax6 and Hes1, and the proliferation marker Ki67 (Fig. 5A-D).
The majority of the cells with the basal processes express Pax6 $(87.5 \pm 5.2 \%)$ and $\mathrm{Ki} 67(84.6 \pm 6.5 \%)$ in the control slices. In the LGN-C-expressing brain, $91.7 \pm 5.2 \%$ of these cells expressed Pax6 and $85.6 \pm 6.0 \%$ expressed Ki67 (Fig. 5D), suggesting that the majority of EGFP-expressing cells with basal processes are proliferative neural progenitors. Moreover, more than two thirds of these cells expressed Hes1 $(67.0 \pm 8.4 \%$ in the control and $78.5 \pm 3.9 \%$ in the LGN-C-expressing cells) (Fig. 5D). Thus, Notch is activated in the majority of these cells, suggesting that they are in an undifferentiated state. We also underwent the same set of experiments using LGN siRNA to suppress LGN function and obtained essentially similar results (supplemental Fig. S3, available at www.jneurosci.org as supplemental material). By staining with an anti-phospho-vimentin antibody, which marks mitotic cells, we confirmed that these proliferating cells outside of the $\mathrm{VZ}$ retain their basal processes during mitosis (supplemental Fig. S4, available at www.jneurosci.org as supplemental material) (Ochiai et al., 2009). These observations of fixed sections led us to conclude that outer $\mathrm{VZ}$ progenitors with the basal process are indeed formed in the normal developing brain (Fig. 4). We next examined the temporal profile of the proportion of the outer $\mathrm{VZ}$ progenitors in the fixed sections (supplemental Fig. S5, available at www.jneurosci.org as supplemental material). The number of Pax6/Hes1 double-positive cells outside of the VZ gradually increased from E15.5 to E18.5 [7.8 \pm 2.7 per unit columnar region of cortex (100 $\mu \mathrm{m}$ wide) at E15.5, $14.4 \pm 2.6$ at E16.5, and $20.2 \pm$ 0.9 at E18.5]. The percentage of Pax6/Hes1 double-positive cells outside of the VZ increased more drastically from $6.0 \pm 1.7 \%$ at E15.5 to $34.3 \pm 0.8 \%$ at E18.5, because the number of Pax6/Hes 1 double-positive cells within the VZ greatly reduced from $116.0 \pm$ 8.7 per unit columnar region of cortex at E16.5 to $38.8 \pm 2.6$ at E18.5. We concluded that there is a minor population of outer VZ progenitors that self-renew in the developing mouse brain, and these cells retain only their basal processes. Because they are increased by perturbing the orientation of the cleavage plane, it is highly likely that such cells are formed by their failure to inherit the apical junction after oblique cleavage.

\section{Clonal Notch-Delta signaling is essential for the maintenance of the outer $\mathrm{VZ}$ progenitors}

In the VZ, the daughter cells from the apical divisions interact with each other and also with differentiating neighbor cells that were born from other apical daughters via Notch-Delta signaling (Yoon et al., 2008). Nascent basal progenitors and neurons in the $\mathrm{VZ}$ are the main cell types that express a major Notch ligand, Delta-like1, and a Delta regulator, Mindbomb (Kawaguchi et al., 2008). These facts raise the question of how the outer VZ progenitors receive Notch ligands. One possibility is that the daughter cells of the outer VZ progenitors are the source of Delta. To test this, we attempted to eliminate Delta function in the lineage of the outer VZ progenitors by coexpressing a dominant-negative form of Mindbomb-1 with LGN-C in the developing mouse brain. Mib1 is an E3 ubiqutin ligase, which is essential for endocytosis of Delta proteins and Notch activation. The Mib1 mutant lacking its ring finger domain acts as a dominant-negative (DNMib1) (Zhang et al., 2007). If Notch ligands are mainly provided by the two sibling cells during the formation and maintenance of the outer $\mathrm{VZ}$ progenitors, then the expression of DN-Mib1 with LGN-C should compromise Notch-Delta signaling between the daughter cells and result in a failure to form outer VZ progenitors (Fig. 6A). We examined Pax6 expression in the cells outside of the VZ. As previously shown (Konno et al., 2008), the elimination of LGN function (by overexpression of LGN-C) significantly in- 
A E15 Control

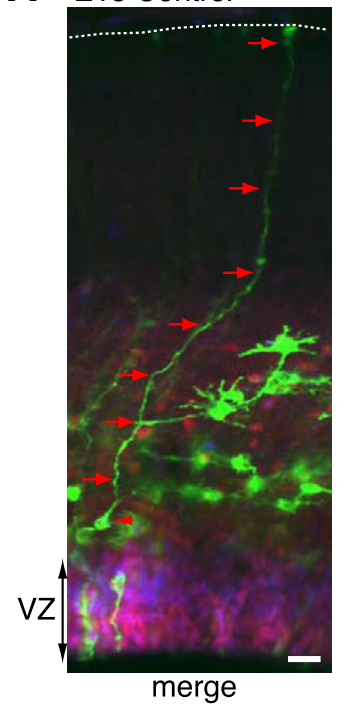

C

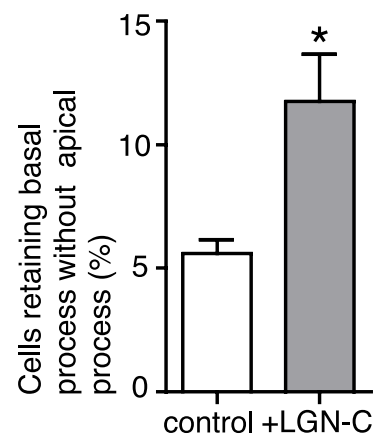

B

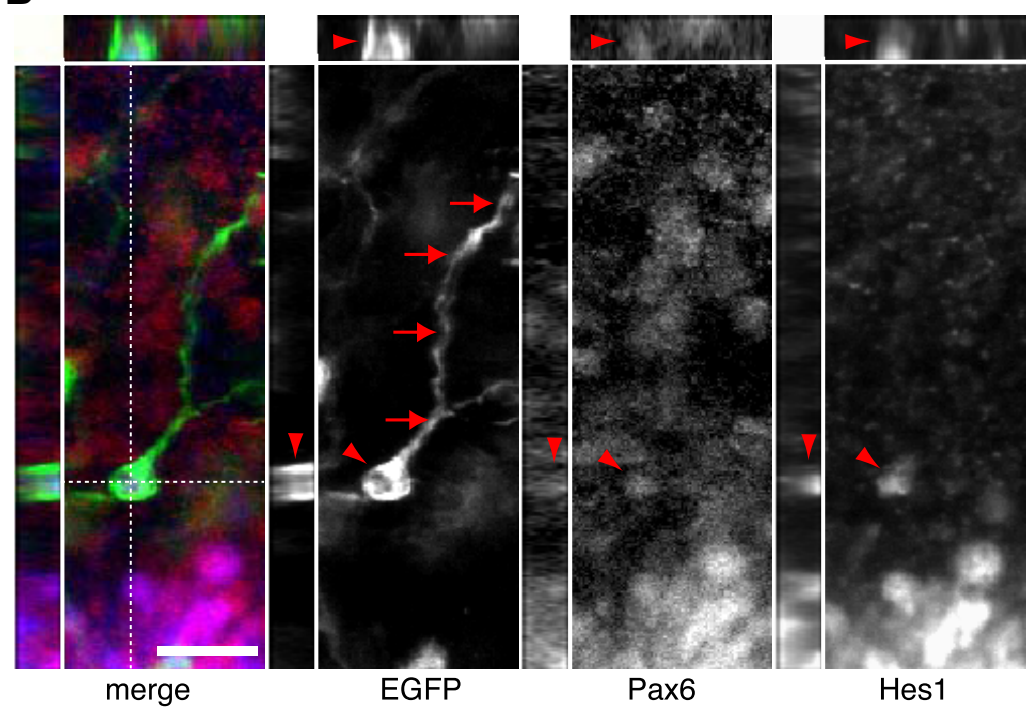

D

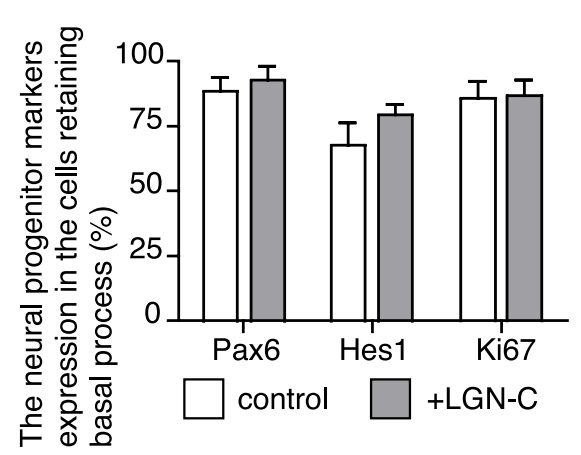

E

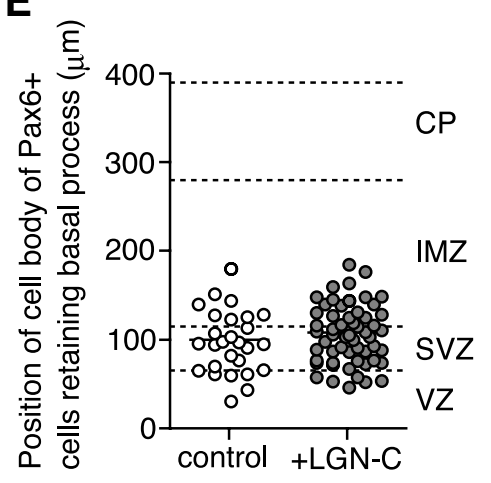

Figure 5. Characterization of the outer VZ progenitor in vivo. $\boldsymbol{A}, \boldsymbol{B}$, The classification of the cells by morphology and neural progenitor marker expression. The cell morphology is visualized by EGFP staining expressed by the F-loxP-EGFP conditional expressing system and vibratome section staining. The brains were electroplated with the plasmids for Cre ( $0.1 \mathrm{ng} / \mu \mathrm{l}$ ) and FloxP-EGFP ( 0.5 $\mu \mathrm{g} / \mu \mathrm{l})$ at E13.5 and fixed to stain for Pax6 and Hes $148 \mathrm{~h}$ later. The cell morphology was analyzed by 3D-reconstructed images of EGFP staining. $\boldsymbol{A}$, the single plane image that contains the entire length of the basal process of a outer VZ progenitor in the 3D-reconstructed image of a control wild-type brain (30 Z-sections with $1.5 \mu \mathrm{m}$ intervals). Arrowhead indicates the cells that retained the basal process but not the apical process. Arrows indicate the basal process that extended to the pial surface. The dashed line indicates the pial surface. Scale bar, $20 \mu \mathrm{m}$. $\boldsymbol{B}$, The magnification of XY sections of the cell that retained the basal process shown in $\boldsymbol{A}$. Arrows indicate the basal process, and arrowheads indicate the cell body. Scale bar, $10 \mu \mathrm{m}$. $\boldsymbol{C}$, $\mathrm{Quantification} \mathrm{of} \mathrm{the} \mathrm{cells} \mathrm{that} \mathrm{retained}$ the basal process but not the apical process. A small number of these cells is observed in the normal brain ( $5.6 \pm 0.6 \%$ of EGFP-positive cells, $n=576$ for three brains), and LGN-C overexpression significantly increased this population (11.8 $\pm 1.9 \%$ of EGFP-positive cells, $n=572$ for four brains). Student's $t$ test, $\left.{ }^{*} p=0.04\right)$. $D, 0$ uantified result of the percentage of the cells that expressed Pax6, Hes1, or Ki67 in the cells that retained the basal process without the apical process in the wild-type control (Pax6 87.5 $\pm 5.2 \%, n=56$ for six brains; Hes $167.0 \pm 8.4 \%, n=34$ for four brains; Ki67 84.6 $\pm 6.5 \%, n=40$ for four brains) and on overexpression of LGN-C (Pax691.7 $\pm 5.2 \%, n=62$ for four brains; Hes $178.5 \pm 3.9 \%, n=41$ for four brains; Ki67 85.6 $\pm 6.0 \%, n=31$ for four brains). $\boldsymbol{E}$, Position of the cell bodies of Pax6-expressing cells that retained the basal process without the apical process. The boundary between the VZ and SVZ was defined as the position where the density of Pax6-positive cells drastically dropped. The boundary of the SVZ and outer SVZ was similarly determined as the basal border of the region containing a high density of Tbr2-positive cells.

creased the percentage of Pax6-positive cells outside of the VZ (Fig. 6C, $4.2 \pm 0.5 \%$ in the control and $13.2 \pm 1.6 \%$ in LGN-C overexpression), while decreasing their percentage within the $\mathrm{VZ}$ (Fig. $6 D, 32.1 \pm 2.5 \%$ in the control and $23.5 \pm 2.0 \%$ in LGN-C overexpression). The simultaneous expression of DN-Mib1 with LGN-C did not change the percentage of Pax6-positive cells in the $\operatorname{VZ}(23.9 \pm 1.3 \%)$ (Fig. $6 D)$. A similar result was obtained when Mib1 function was eliminated in a single lineage of apical progenitors using conditional Mibl-knock-out (KO) mice (Yoon et al., 2008). These results indicate that the loss of Mib1 does not affect the apical progenitor cell autonomously, and that the loss of Delta function in the sibling cell has no effect on the apical progenitor, implying that Notch ligands are presented to the apical progenitors by not only their daughters but also surrounding differentiating cells in the VZ. In contrast, the percentage of Pax6-positive cells outside of the VZ was significantly reduced by the coexpression of DN-Mib1 (6.3 $\pm 0.4 \%$ ) (Fig. 6C). These results suggest that the survival of the outer VZ progenitors is not sufficiently supported by the supply of Notch ligands in the $\mathrm{VZ}$, which is effective for the apical progenitors, but that the outer $\mathrm{VZ}$ progenitors require their progeny cells as a source of Notch ligands. We further investigated whether Notch ligands are clonally provided to the outer VZ progenitors. To address this point, we examined the effect of DN-Mib1 by using time-lapse imaging at the single-cell level under clonally isolated conditions. When DN-Mib1 and LGN-C are coexpressed by the Cre-loxP clonal expression plasmid system (Morin et al., 2007), we observed the formation of an outer VZ progenitor in only $2.5 \pm 2.5 \%$ (Fig. 7), which is in contrast to the frequent formation of outer $\mathrm{VZ}$ progenitors when LGN-C single expression is used (Fig. 4, 43.7 \pm 6.3). These data strongly suggest that the basal daughter cells generated by oblique divisions do not self-renew if Delta-Notch 
signaling is clonally compromised. We do not exclude the possibility that this defect may have been caused by some yetunidentified form of abnormal signaling that is specifically required for these types of cells and unnecessary for the apical progenitors. The outer VZ progenitors are thus most likely to depend on Notch ligands from their daughter cells. In contrast, the coexpression of DN-Mib1 and LGN-C showed no apparent effect on the apical progenitors within the VZ (Fig. 7B$D)$, indicating that the sibling cell is dispensable as a provider of Notch ligands for the apical progenitors, as shown in a previous study (Yoon et al., 2008). We conclude that outer $\mathrm{VZ}$ progenitors are formed and maintained by clonal NotchDelta signaling, where Delta ligands are supplied by their sibling cells.

\section{Discussion}

The roles of epithelial structures in neural progenitor self-renewal

In this study, time-lapse imaging analysis revealed that self-renewing progenitors in the VZ (apical progenitors) are derived from daughter cells that inherit the complete epithelial structure as a result of apical divisions during the mid-neurogenic stage. This mode of division is different from that observed in the zebrafish hindbrain (Alexandre et al., 2010) or chicken spinal cord (Wilcock et al., 2007), in which the cleavage plane frequently bypasses the apical membrane to generate a more basal daughter cell and a more apical daughter cell. The more basal daughter cell in the zebrafish hindbrain restores the epithelial structure by regenerating the apical process and selfrenewing. Thus, the complete epithelial structure is likely to be important for the self-renewal of apical progenitors, regardless of the division mode, whereas the division mode of self-renewing progenitors depends on the developmental timing and context.

Here, we showed that in the developing mouse brain oblique cleavage plane orientations that bypass the apical surface indeed generate the more basal self-renewing daughter cells and the more apical daughter cells that commit to differentiation, although the frequency of such divisions is low in vivo. This combination of daughter cell fates is consistent with an observation in the zebrafish hindbrain described above (Alexandre et al., 2010), but the behavior of the self-renewing basal daughter cells is quite different from that of the basal daughter cells in the zebrafish. The basal daughter cells in the mouse brain migrate out of the VZ without regeneration of the apical endfoot and undergo asymmetric divisions as outer VZ progenitors that retain their basal process (or become basal progenitors). These observations are consistent with our represents SEM.
A

B
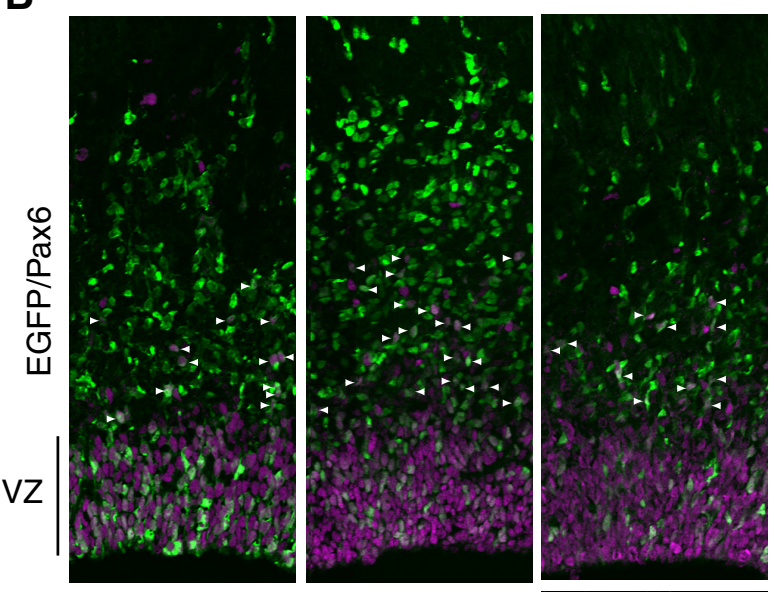

C
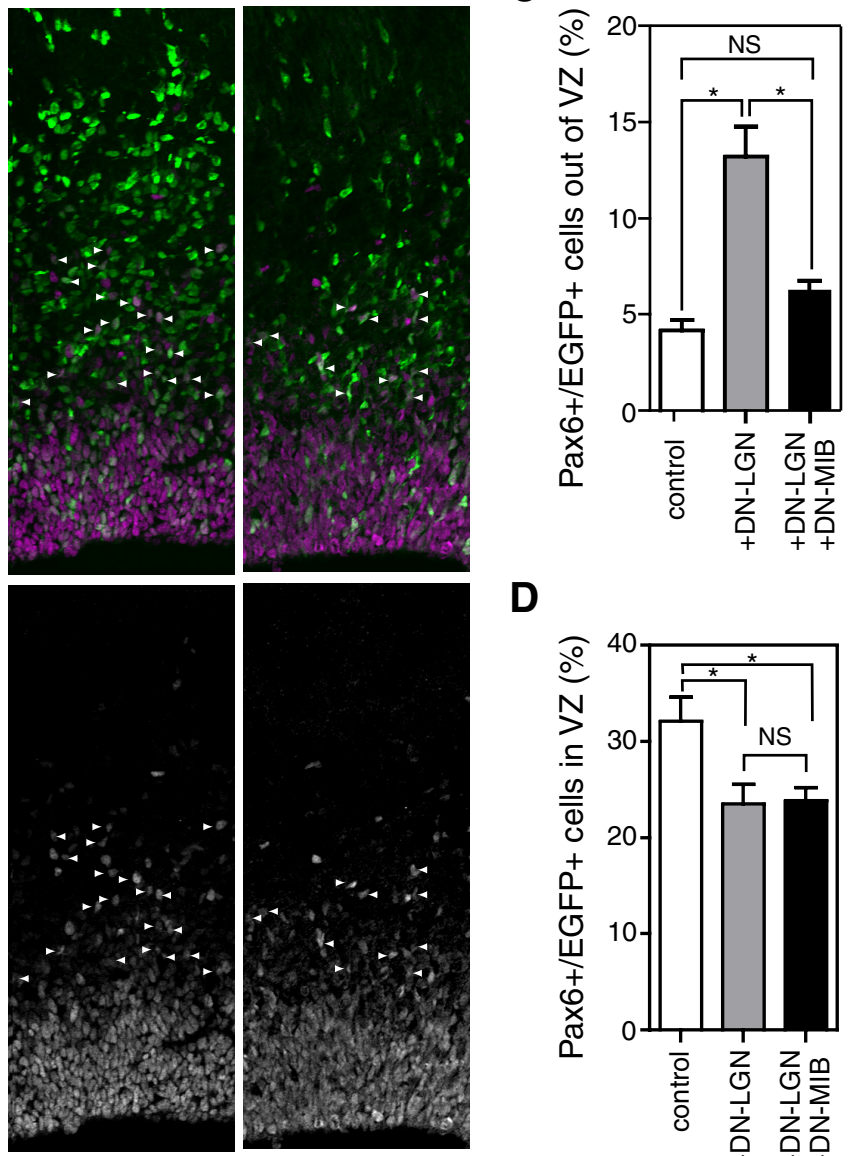

$+\mathrm{DN}-\mathrm{LGN}$
D

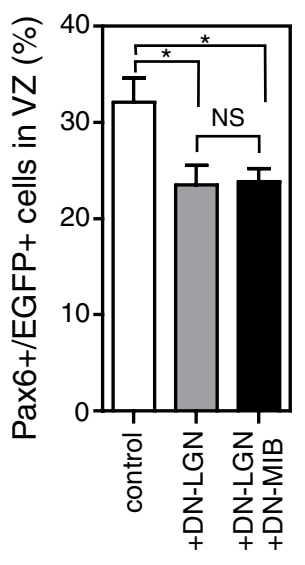

Figure 6. Overexpression of dominant-negative Mindbomb-1 suppresses the Pax6-positive outer VZ progenitors induced by spindle misorientation. $A$, Simplified drawing of the effect of the DN-Mib1. The overexpression of DN-Mib1 inhibits signaling from Delta-expressing cells. $\boldsymbol{B}$, The brains were electroporated with the plasmids for EGFP-3NLS $(0.2 \mu \mathrm{g} / \mu \mathrm{l})$, empty vector $(1.5 \mu \mathrm{g} / \mu \mathrm{l})$, LGN-C $(1.5 \mu \mathrm{g} / \mu \mathrm{l})$, and DN-Mib1 $(1.5 \mu \mathrm{g} / \mu \mathrm{l})$ (in the indicated combinations) at E13.5, and was analyzed $48 \mathrm{~h}$ later. The fixed brain sections were used to visualize the electroporated cells by EGFP staining and to reveal the undifferentiated progenitors by Pax6 staining. The white arrowheads in the top and bottom panels indicate the Pax6/EGFP double-positive cells outside of the VZ. Scale bar, $20 \mu \mathrm{m}$. C, Quantified results of the Pax6/EGFP double-positive cells outside of the VZ. LGN-C overexpression significantly increased the number of Pax6-positive cells outside of the VZ, and this effect is significantly suppressed by DN-Mib1 overexpression. One-way ANOVA with post-Bonferroni multiple-comparison test, ${ }^{*} p<0.001$. NS, Not significant. Five embryos were analyzed per sample. Error bar represents SEM. D, Quantified results of the Pax6/EGFP double-positive cells in the VZ. LGN-C overexpression significantly decreased the number of Pax6-positive cells in the VZ, whereas DN-Mib1 overexpression had no effect. One-way ANOVA with post-Bonferroni multiple-comparison test, ${ }^{*} p<0.05$. Five embryos were analyzed per sample. Error bar

previous study on LGN KO mouse, in which the apical progenitors were transformed into Pax6-positive mitotic cells outside of the SVZ without affecting the overall neuronal production rate (Konno et al., 2008).

The invariant inheritance of the basal process by the selfrenewing daughter cell both within and outside of the VZ suggests essential roles of the basal process in progenitor self-renewal. One possibility is that the basal process may transmit signals that are 
A E14 LGN-C + DN-Mib1 overexpression Basal progenitor/Neuron (post-mitotic)
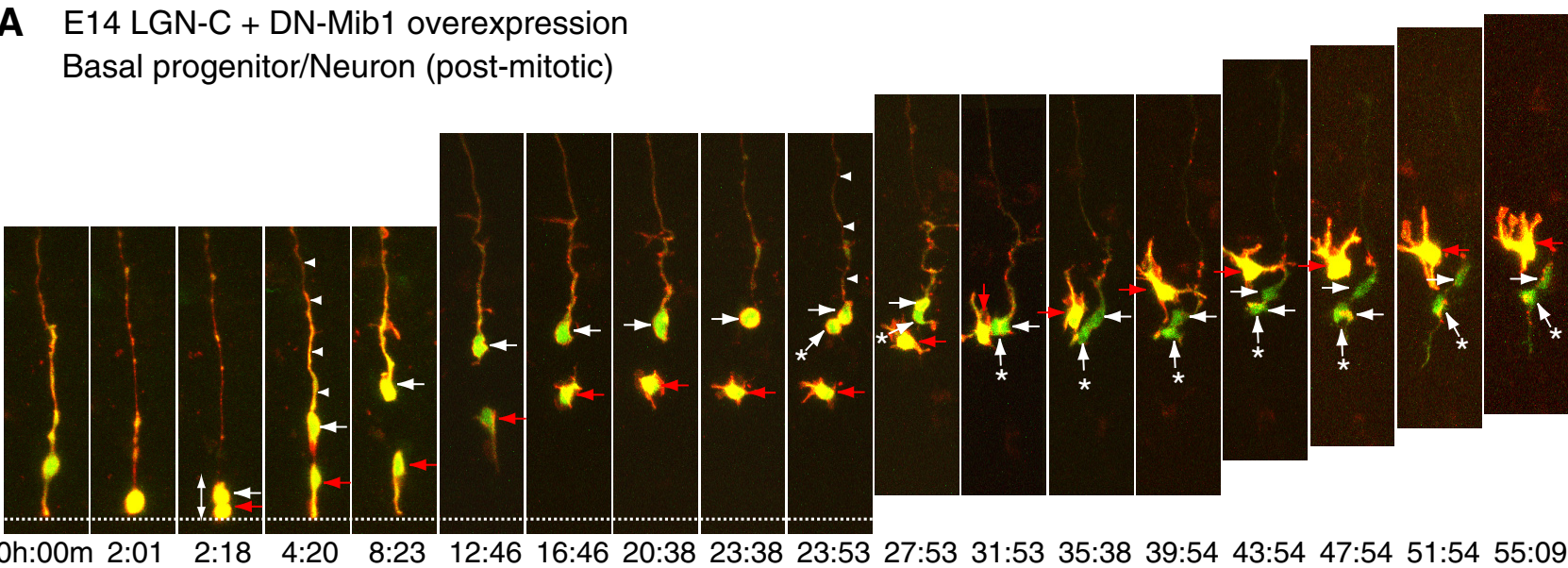

\section{B Basal process inheritance}

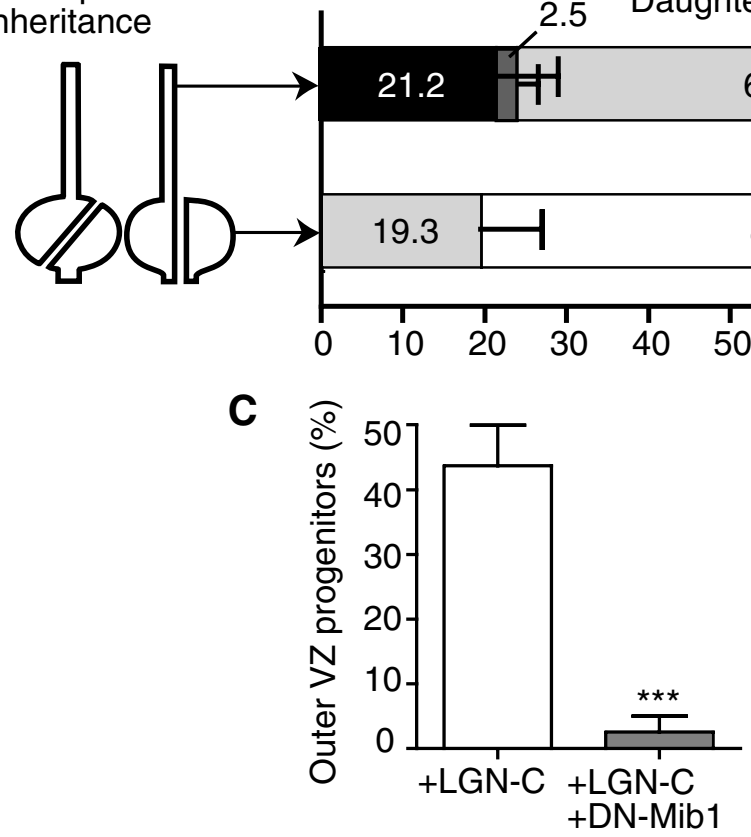

\section{27:53 31:53 35:38 39:54 43:54 47:54 51:54 55:09}


2010). This lack of correlation does not necessarily indicate that the apical junction is dispensable for self-renewal but raises the possibility that the function of the basal process may need to be coupled with these apically mediated signals for the daughters formed from apical divisions to acquire self-renewability. This accounts for the correlation of the entire epithelial structure with self-renewal. Outer VZ progenitors lack the apical junction but may form transient local adhesions between sibling cells, which may compensate for the loss of the apical junction during division.

\section{The asymmetric inheritance of apical junctions is a mechanism for generating self-renewing progenitors outside of the germinal zone in the mouse brain}

Oblique apical divisions efficiently induce self-renewing outer $\mathrm{VZ}$ progenitors by causing the lack of the apical endfoot in one daughter cell and not affecting the overall neuronal production rate, as shown by time-lapse imaging in this study and by the analysis of LGN mutant phenotypes (Konno et al., 2008). This is in contrast to cells delaminating in the VZ by the retraction of the apical process during interphase, because the delamination of the apical progenitor is normally coupled with neural differentiation (Ge et al., 2006). The acute loss of the apical junction, as well as the disruption of cell polarity in a single cell, also promotes neuronal differentiation (Costa et al., 2008; Bultje et al., 2009; Zhang et al., 2010), while the loss of polarity en masse leads to a gross disruption of the cortical tissue structure (Lien et al., 2006). These observations indicate that self-renewing progenitors that have lost cell polarity or adhesion are difficult to delaminate from the epithelial structure with maintenance of the undifferentiated state. Thus, the asymmetric inheritance of the apical junction during apical divisions appears to be a key process for the generation of self-renewing progenitors outside of the VZ.

\section{Mouse outer VZ progenitors share cell properties with OSVZ progenitors in gyrencephalic mammals}

OSVZ progenitors are a different type of self-renewing progenitors implicated in cortical development in the primate and other gyrencephalic mammals (Kriegstein et al., 2006; Dehay and Kennedy, 2007). They emerge at the mid-neurogenic stage and are distributed outside of the ordinary germinal zone (the VZ and the SVZ). Because the OSVZ progenitors are the major type of neural progenitors from mid-neural development onward (the period of primate brain expansion), their emergence has been implicated in brain expansion during mammalian evolution. Although the properties of these OSVZ progenitors are unclear, recent studies using human embryos and ferrets report that these progenitors are distributed outside of the SVZ and often lose the apical process (Fietz et al., 2010; Hansen et al., 2010) but retain the basal process (Kriegstein et al., 2006; Fish et al., 2008). These properties are exactly the same as the ones we observed in the outer VZ progenitors, as follows: (1) forms outside of the VZ to eventually distribute outside of the SVZ, as observed in the LGN KO mice; (2) retains the basal process that reaches the basal laminar but not the apical process; (3) expresses the Pax6 marker for self-renewing progenitors; and (4) requires Notch activation for self-renewal and, hence, expresses Hes1. Therefore, we propose that the outer VZ progenitors in the mouse are the mouse counterparts of the OSVZ progenitors observed in gyrencephalic mammals such as primates. If this is the case, then asymmetric segregation of the apical junction due to the oblique divisions is a likely mechanism of generating OSVZ progenitors in gyrencephalic mammals. In human and ferret embryos, the majority of apical progenitors display cleavage planes nearly vertical to the epithelial plane (Fietz et al., 2010; Hansen et al., 2010) (our unpublished data), as observed in the mouse (Konno et al., 2008). This suggests that the formation of OSVZ progenitors occurs at a low frequency, hence taking time for the accumulation of these cells. This prediction is consistent with the appearance of OSVZ progenitors at mid-neurogenic stages after a latent period. In rats, self-renewing progenitors have not been detected (Noctor et al., 2008). We believe that the behavior of mouse outer VZ progenitors, at least in part, mimics the process of the formation and/or the maintenance of the gyrencephalic OSVZ progenitors. Thus, outer VZ progenitors induced by oblique cleavage will provide a convenient model system to study the properties of OSVZ progenitors in gyrencephalic mammals.

While mouse outer $\mathrm{VZ}$ progenitors are quite similar to OSVZ progenitors in gyrencephalic mammals, there is a considerable difference in the progress of neurogenesis between mice and primates (and ferrets). In primates and ferrets, the population of OSVZ progenitors extensively expands to make the OSVZ the major germinal zone from the mid-neurogenic stage onward. This is in contrast to the mouse, in which the progenitor population rapidly declines and neurogenesis is soon terminated, while the proportion of the outer VZ progenitors to the entire population of self-renewing progenitors gradually increases (up to $30 \%$ at E18). This may be due to the difference in the intrinsic cell state of OSVZ progenitors and/or because the gyrencephalic OSVZ provides a niche that confers more self-renewability to cells.

In summary, this study demonstrates that the relationship between progenitor self-renewal and the basal process is a common feature in neural stem cells in the developing brain. Our approach, using induced spindle misorientation in the mouse neocortex, provides an excellent strategy to study not only progenitor self-renewal but also OSVZ progenitors in gyrencephalic mammals. Based on our results, we propose a working hypothesis that the loss of apical junctions during oblique apical division is a mechanism for generating gyrencephalic OSVZ progenitors.

\section{References}

Alexandre P, Reugels AM, Barker D, Blanc E, Clarke JD (2010) Neurons derive from the more apical daughter in asymmetric divisions in the zebrafish neural tube. Nat Neurosci 13:673-679.

Bultje RS, Castaneda-Castellanos DR, Jan LY, Jan YN, Kriegstein AR, Shi SH (2009) Mammalian Par3 regulates progenitor cell asymmetric division via notch signaling in the developing neocortex. Neuron 63:189-202.

Campos LS, Decker L, Taylor V, Skarnes W (2006) Notch, epidermal growth factor receptor, and beta 1-integrin pathways are coordinated in neural stem cells. J Biol Chem 281:5300-5309.

Costa MR, Wen G, Lepier A, Schroeder T, Götz M (2008) Par-complex proteins promote proliferative progenitor divisions in the developing mouse cerebral cortex. Development 135:11-22.

Dehay C, Kennedy H (2007) Cell-cycle control and cortical development. Nat Rev Neurosci 8:438-450.

Fietz SA, Kelava I, Vogt J, Wilsch-Bräuninger M, Stenzel D, Fish JL, Corbeil D, Riehn A, Distler W, Nitsch R, Huttner WB (2010) OSVZ progenitors of human and ferret neocortex are epithelial-like and expand by integrin signaling. Nat Neurosci 13:690-699.

Fish JL, Dehay C, Kennedy H, Huttner WB (2008) Making bigger brainsthe evolution of neural-progenitor-cell division. J Cell Science 121:2783-2793.

Gaiano N, Nye JS, Fishell G (2000) Radial glial identity is promoted by Notch 1 signaling in the murine forebrain. Neuron 26:395-404.

Ge W, He F, Kim KJ, Blanchi B, Coskun V, Nguyen L, Wu X, Zhao J, Heng JI, Martinowich K, Tao J, Wu H, Castro D, Sobeih MM, Corfas G, Gleeson JG, Greenberg ME, Guillemot F, Sun YE (2006) Coupling of cell migration with neurogenesis by proneural bHLH factors. Proc Natl Acad Sci U S A 103:1319-1324.

Hansen DV, Lui JH, Parker PR, Kriegstein AR (2010) Neurogenic radial glia 
in the outer subventricular zone of human neocortex. Nature 464:554-561.

Hartfuss E, Galli R, Heins N, Götz M (2001) Characterization of CNS precursor subtypes and radial glia. Dev Biol 229:15-30.

Kato TM, Kawaguchi A, Kosodo Y, Niwa H, Matsuzaki F (2010) Lunatic fringe potentiates Notch signaling in the developing brain. Mol Cell Neurosci 45:12-25.

Kawaguchi A, Ikawa T, Kasukawa T, Ueda HR, Kurimoto K, Saitou M, Matsuzaki F (2008) Single-cell gene profiling defines differential progenitor subclasses in mammalian neurogenesis. Development 135:3113-3124.

Knoblich JA (2008) Mechanisms of asymmetric stem cell division. Cell 132:583-597.

Konno D, Shioi G, Shitamukai A, Mori A, Kiyonari H, Miyata T, Matsuzaki F (2008) Neuroepithelial progenitors undergo LGN-dependent planar divisions to maintain self-renewability during mammalian neurogenesis. Nat Cell Biol 10:93-101.

Kosodo Y, Röper K, Haubensak W, Marzesco AM, Corbeil D, Huttner WB (2004) Asymmetric distribution of the apical plasma membrane during neurogenic divisions of mammalian neuroepithelial cells. EMBO J 23:2314-2324.

Kriegstein A, Noctor S, Martínez-Cerdeño V (2006) Patterns of neural stem and progenitor cell division may underlie evolutionary cortical expansion. Nat Rev Neurosci 7:883-890.

Lien WH, Klezovitch O, Fernandez TE, Delrow J, Vasioukhin V (2006) alpha E-catenin controls cerebral cortical size by regulating the hedgehog signaling pathway. Science 311:1609-1612.

Manabe N, Hirai S, Imai F, Nakanishi H, Takai Y, Ohno S (2002) Association of ASIP/mPAR-3 with adherens junctions of mouse neuroepithelial cells. Dev Dyn 225:61-69.

Miyata T, Kawaguchi A, Okano H, Ogawa M (2001) Asymmetric inheritance of radial glial fibers by cortical neurons. Neuron 31:727-741.

Miyata T, Kawaguchi A, Saito K, Kawano M, Muto T, Ogawa M (2004) Asymmetric production of surface-dividing and non-surface-dividing cortical progenitor cells. Development 131:3133-3145.

Morin X, Jaouen F, Durbec P (2007) Control of planar divisions by the G-protein regulator LGN maintains progenitors in the chick neuroepithelium. Nat Neurosci 10:1440-1448.

Noctor SC, Flint AC, Weissman TA, Dammerman RS, Kriegstein AR (2001) Neurons derived from radial glial cells establish radial units in neocortex. Nature 409:714-720.
Noctor SC, Martínez-Cerdeño V, Ivic L, Kriegstein AR (2004) Cortical neurons arise in symmetric and asymmetric division zones and migrate through specific phases. Nat Neurosci 7:136-144.

Noctor SC, Martínez-Cerdeño V, Kriegstein AR (2008) Distinct behaviors of neural stem and progenitor cells underlie cortical neurogenesis. J Comp Neurol 508:28-44.

Ochiai W, Nakatani S, Takahara T, Kainuma M, Masaoka M, Minobe S, Namihira M, Nakashima K, Sakakibara A, Ogawa M, Miyata T (2009) Periventricular notch activation and asymmetric Ngn2 and Tbr2 expression in pair-generated neocortical daughter cells. Mol Cell Neurosci 40:225-233.

Shioi G, Konno D, Shitamukai A, Matsuzaki F (2009) Structural basis for self-renewal of neural progenitors in cortical neurogenesis. Cereb Cortex 19:i55-61.

Suzuki Y, Yanagisawa M, Yagi H, Nakatani Y, Yu RK (2010) Involvement of beta 1-integrin up-regulation in basic fibroblast growth factor- and epidermal growth factor-induced proliferation of mouse neuroepithelial cells. J Biol Chem 285:18443-18451.

Wang X, Tsai JW, Imai JH, Lian WN, Vallee RB, Shi SH (2009) Asymmetric centrosome inheritance maintains neural progenitors in the neocortex. Nature 461:947-955.

Wilcock AC, Swedlow JR, Storey KG (2007) Mitotic spindle orientation distinguishes stem cell and terminal modes of neuron production in the early spinal cord. Development 134:1943-1954.

Yoon K, Gaiano N (2005) Notch signaling in the mammalian central nervous system: insights from mouse mutants. Nat Neurosci 8:709-715.

Yoon KJ, Koo BK, Im SK, Jeong HW, Ghim J, Kwon MC, Moon JS, Miyata T, Kong YY (2008) Mind bomb 1-expressing intermediate progenitors generate Notch signaling to maintain radial glial cells. Neuron 58:519-531.

Zhang C, Li Q, Jiang YJ (2007) Zebrafish Mib and Mib2 are mutual E3 ubiquitin ligases with common and specific Delta substrates. J Mol Biol 366:1115-1128.

Zhang J, Woodhead GJ, Swaminathan SK, Noles SR, McQuinn ER, Pisarek AJ, Stocker AM, Mutch CA, Funatsu N, Chenn A (2010) Cortical neural precursors inhibit their own differentiation via $\mathrm{N}$-cadherin maintenance of beta-catenin signaling. Dev Cell 18:472-479. 